\title{
The Connection between Gravity and Electricity According to Twin Physics and a Survey of the Results So Far, Including Neutron Decay
}

\author{
Anna C.M. Backerra ${ }^{1}$ \\ ${ }^{1}$ Gualtherus Sylvanusstraat 2, 7412 DM Deventer, The Netherlands \\ Correspondence: Anna C.M. Backerra, Gualtherus Sylvanusstraat 2, 7412 DM Deventer, The Netherlands. \\ E-mail: annabackerra@gmail.com
}

Received: November 7, 2016

Accepted: November 18, 2016 Online Published: November 28, 2016

doi:10.5539/apr.v8n6p42

URL: http://dx.doi.org/10.5539/apr.v8n6p42

\begin{abstract}
Unlike electricity, gravity is active in only one direction, namely attraction. If both phenomena are considered using the mathematical description called complementary language, the range of gravity turns out to be larger than the range of electricity. This seems to be the reason for the apparent one-sidedness of gravity. Beyond the reach of electromagnetic effects, repulsion of physical bodies is possible and this can be identified with expansion of the universe. With this last piece of the puzzle, our dualistic way of considering the universe, called twin physics, seems in principle to be completed. A coherent survey of the results so far is given, including a possible description of neutron decay. The results are compared with the features of the Standard Model.
\end{abstract}

Keywords: gravity, electricity, expanding universe, complementarity, twin physics, neutron decay, neutrino

\section{Introduction}

This is the fifth paper in a series exploring the power of twin physics (Backerra, 2010, 2012, 2014, 2015), a way of considering the universe based upon complementary mathematical language, describing determinate as well as indeterminate aspects of phenomena. Twin physics unites three new principles deviating from classical physics, one philosophical, one mathematical and one physical.

The philosophical principle can be simply expressed as 'every thing has its own nothing', saying that a physical object is not only characterized by its presence in terms of mass, geometry and electromagnetic features, but also by its absence, which is reflected by a finite space around the object, being an independent physical item to which energy is ascribed. The concept of space developed from the classical Greek notion of ether as an extremely thin substance filling the universe, to the assumption of Newton (1687) that space is empty, invisible, unmoving and having an infinite extension. Subsequent scientists changed their minds several times about this notion, until Michelson and Morley (1887), who were unable to find an experimental difference between the velocity of light moving perpendicular to the track of the earth and light moving parallel to it. Since then, space has been considered to be empty and unbounded. By contrast, twin physics considers space as a finite physical object, containing energy and occurring free as well as bounded to a particle.

The mathematical principle underlying complementary language is the joint description of determinate and indeterminate mathematical items in sets. These sets contain not only algebraic but geometric expressions as well, such as spheres, which is unusual. According to Steven Weinberg (2015), geometry played the main role in the brilliant Greek mathematics of the third century BC, whereas algebra was far less developed. By contrast, the modern physicist tends to derive geometric facts algebraically even when geometry is important. Geometry and algebra can be combined by using set theory (Kahn, 1967).

The physical principle is a renewed conception of potential energy. This term was introduced in the 19th century (Rankine, 1853), but it has links to Greek philosopher Aristotle's concept of potentiality. It is conceived as the energy possessed by a body by virtue of its position relative to others, the stresses within itself, its electrical charge, and other factors. Potential energy is associated with the forces that act on a body in a way that depends only on the body's position in space. These forces can be represented by a vector at every point in space forming a vector field of forces. However, potential energy can never be observed in reality because if it is observed, it is no longer potential energy, but actual energy. Twin physics is restricted to the description of physical observations and for that reason we consider potential energy as a mathematical item to deal with the 
transformation of energy from one type into another. To express this basic difference between a mathematical and physical concept of energy, we have introduced the term 'actual energy'. Consequently, the law of conservation of energy is expressed in twin physics as the law of conservation of actual energy.

These three principles deviate considerably from current concepts in physics, resulting in a completely different theoretical structure. Instead of considering elementary particles as the building blocks of the universe, the Heisenberg unit (H-unit), being a unit of potential energy, is introduced as fundamental. Thus, a mathematical item is taken as the starting point instead of a physical phenomenon. The H-unit manifests itself by interaction with another H-unit, as a result of which potential energy is converted into actual energy. Because not necessarily all potential energy is used by a specific interaction, the potential energy of a H-unit acts as an upper boundary for the generation of actual energy. This leads to an upper boundary for the mass of elementary particles instead of a lower boundary, the inverse of the traditional approach, which was to search for the smallest particles in existence.

At the outset of twin theory we constructed a set of four elements called the zipper, a formula collecting the descriptions of all possible physical observations, but it was not clear yet how these results could be identified. We started out rather intuitively, suggesting as a first trial sometimes identifications for specific cases which later turned out to be impossible. One example is the electron, which we had previously thought could be described by the first element of the zipper (Backerra, 2012, section VII.A); later it became clear that only heavy particles like neutrons and protons can be described by this zip. A second recent example is that we are only now certain that a zipper of the first order, containing eight zips, does not describe more observations than a zipper of the second order, containing only four zips, so from now on we will use only zippers of the second order.

It was only after years of laborious jigsaw-puzzling that the relationship between the set elements of the zipper and the manifold of possible observations became comprehensible and coherent. We were encouraged by our success in describing gravity (Backerra, 2014 section IV) and deriving the radius of a proton using its mass, the equivalence of mass and energy and the energy balance between particle and waves (Backerra, 2014 section III.A), as first proof that twin theory describes real physics. It was even more encouraging to be able to describe a photon (Backerra, 2015, section 8), including the quantization of its energy and its constant velocity, without using a postulate. Because the deduction of the photon was based upon both large- and small-scale physics, this description represented a bridge between the two.

It seems appropriate to compare our results with those of the Standard Model, to find out whether it might be an addition or perhaps even a viable alternative. Before investigating this, however, we would like to clear up one intriguing detail about gravity. In a previous paper (Backerra, 2014), we explained how gravity can be described by twin physics, but without considering electricity. At that point, we didn't answer the question why gravity leads only to accelerated attraction between masses, whilst electricity leads to accelerated attraction or repulsion between charged masses. What could be the reason for the restriction of gravity to only one of these two apparently obvious possibilities? This question has occupied many scientists since the discovery of electricity, but we could not find any theories explaining this typical difference between gravity and electricity. Using our results from 2014, we now will take the next step by considering both phenomena together.

After that, we will survey all of the theoretical results obtained so far using twin physics. In the previous four papers we have improved our definitions step by step, resulting in changes of interpretations, some of them drastic. Each time we have presented rather long-winded repetitions of our theory in order to explain these adaptations. This time, only a slight adaptation in the transformation formulas for space attributes in section 2.4 was necessary, to improve the description of the electron. We will now repeat our final definitions and formulas once more, this time as briefly as possible, as a 'dry theory', just to have our theoretical instruments at hand. A thorough consideration of the energy of the zipper is added and based upon it, a description of the neutron decay. More information about the underlying ideas can be found in previous publications, indicated when appropriate.

\section{A Manual for the Use of Twin Physics}

Potential energy is defined as the power to interact. An H-unit is an elementary unity of potential energy, expressed in complementary terms; it can manifest itself only by interacting with other H-units. We suppose that an H-unit in an isolated system starts to interact as soon as another H-unit exists in this system. Then the potential energy will partly or fully be converted into actual energy, resulting in the appearance of one or more Heisenberg-events, written as H-events. H-events are physical realities like a particle or a space. We suppose that the conversion of potential energy into phenomena occurs in a complementary way, as a contraction or as an expansion. In our first publication (Backerra, 2010) you can find more about the basics of twin physics. 
The law of conservation of energy is supposed to be valid for interacting H-units. Considering the universe as an isolated system, built up by interacting $\mathrm{H}$-units, this means that the total amount of actual energy, being generated by all $\mathrm{H}$-units together, is constant. Thus if the universe has a certain starting amount of actual energy, an increasing conversion from potential into actual energy by some $\mathrm{H}$-units has to be balanced by the opposite by others.

Because no independent frame of reference is used, any movement of an H-event is only possible with respect to another H-unit, so relativity is incorporated from scratch. To be able to describe physical items with the zipper, we introduced three mathematical qualities: time, space and mark (the last one being the mathematical precursor of electromagnetic features). For each quality, an H-unit is supplied with pairs of complementary mathematical attributes, each pair containing a determinate and an indeterminate attribute.

If we express the Heisenberg's principle as "each observation of certainty implies an amount of uncertainty", an extended Heisenberg's principle can be created by adding the complementary sentence "each observation of uncertainty implies an amount of certainty". This fundamental feature of nature is anchored in our theory by supposing that all attributes add to any observation in pairs, which are restricted by the following axiom: Attributes contribute to an observation in pairs, which are such that one member is of major and the other of minor importance. Therefore an H-unit $H_{i}$ is, for each quality, supplied with a set $h_{i}$ of mathematical attributes in two types: Determinate attributes are represented by $D_{i}$ or $d_{i}$, and indeterminate ones by $U^{i}$ or $u^{i}$; attributes of major importance are indicated in capitals and that of minor importance in lower case. They are collected in the general set of attributes:

$$
h_{i}=\left\{D_{i}, U^{i}, d_{i}, u^{i}\right\}
$$

Each H-unit has a set of four attributes for each quality, so all together it has twelve attributes. The interaction between two $\mathrm{H}$-units $H_{i}$ and $H_{j}$ is written as $H_{i}{ }^{*} H_{j}$. Any interaction is based upon the exchange of attributes, according to mathematical rules which may differ for each quality. By transforming exchanged attributes from mathematical into physical items, potential energy is converted into actual energy, describing phenomena. An H-unit may have no mark attributes; then it is called a neutral H-unit, written as $H_{0}$. A neutral $\mathrm{H}$-unit has the same potential energy as a marked $\mathrm{H}$-unit and so its spatial dimensions are larger.

We defined two operators to describe exchange: the join operator $\triangleright \triangleleft$, pronounced as 'joined with', and the link operator $\propto$, pronounced as 'linked to'. The join operator connects one major with one minor attribute to a joined pair. The members of one pair may be both determinate, both indeterminate or mixed. After transformation into a physical space, joined pairs occur combined in any phenomenon and so are necessarily observed together. The rules acording to which the operation 'joining' is carried out for each quality, will be given after defining time, space and mark attributes. The link operator couples joined pairs of attributes to chains. If joined pairs are linked and transformed, their intersection may represent a physical observation. The link $X \propto Y$ of mathematical objects $X$ and $Y$ is defined as their intersection. If $X \propto Y$, then $Y \propto X$; if $X=Y$, then $X \propto Y=X ; X \propto \varnothing=\varnothing$ in which $\varnothing$ is an empty set.

With these two operators, the interaction between H-units can be defined by collecting all possible combinations of their attributes of one and the same quality, two by two joined to pairs and these pairs linked to each other in chains. There exist 40 distinct chains for each of the three qualities time, space and mark, but this amount reduces drastically by realizing that complementary observations cannot be observed simultaneously, as we have learned from quantum mechanics. This is expressed as the exclusion principle, saying that a joined pair containing a determinate major attribute cannot link with a joined pair containing an indeterminate major attribute of the same quality and the same H-unit.

The two consequences of the exclusion principle are, that chains of more than four joined pairs cannot be transformed into actual H-events, and that only four distinct chains of four joined pairs exist. Smaller chains do not add more information, but they are less practical to work with.

These four chains are not yet enough to define the zipper, because the operators are defined such that, after carrying out the operations joining and linking, the small-scale elements contain only minor attributes, which will lead to only small-scale observations. To be able to describe as well large-scale observations, each chain is stripped down to the participating two major attributes, which will be used along with the original chains. In doing so, each phenomenon will be characterized by a large- as well as a small-scale description. 
Each of the four original chains is, together with its belonging stripped chain, collected in a set called a $z i p$. In this set, the large-scale element (containing only major attributes) is placed at the left hand and the small-scale element (containing both major and minor attributes) at the right hand.

Then the zipper in general, describing the complete interaction $H_{i}{ }^{*} H_{j}$, can be written as the set of four zips in which each zip is a set of two elements:

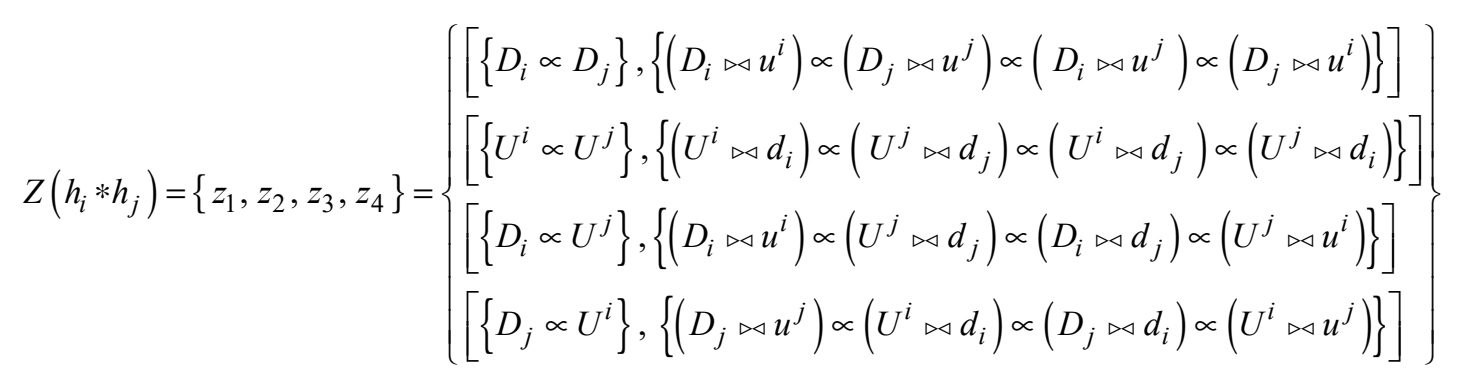

The square brackets around each zip indicate the transformation of mathematical objects into physical items by representing them in a physical space. The indication $h_{i} * h_{j}$ will be dropped later.

After transformation, each zip is a set of two elements, of which the first is the large-scale description and the second the small-scale one. Usually only two of them can be transformed into an H-event. Because of the principle of exclusion, distinct zips cannot be observed simultaneously.

Before transformation, the potential energy of the zipper is equal to the sum of potential energy of the participating H-units, which is twice the potential energy of one H-unit. The potential energy of an H-unit is by definition a unity, which will be indicated by the constant $V$, as a reminiscence to the original definition by Rankine (1853). So after transformation, the actual energy $E$ of the zipper, and thus the actual energy of the interaction can be expressed as:

$$
E\left(H_{i}^{*} H_{j}\right) \leq 2 \times V
$$

The actual energy of the zipper is also supposed to be the sum of the generated actual energies of the four zips:

$$
E\left(H_{i} * H_{j}\right)=\sum_{n=1}^{n=4} E\left(z_{n}\right) .
$$

The potential energies of the zips are supposed to be equal. By transforming a zip, its potential energy will fully, partly or not at all be converted into actual energy, depending on the type of interaction, so actual energies of the zips are not necessarily equal. The potential energy of the complete zipper may be fully, partly or not at all be converted into actual energy. Equations (3) and (4) suggest that the potential energy of one zip is maximum equal to $1 / 2 \times V$; the minimum of actual energy of the complete zipper is by definition zero (if $H_{i}$ and $H_{j}$ have no interaction at all). We suppose that the maximum occurs if $H_{i}$ and $H_{j}$ have coinciding time and space attributes. As we know from previously deduced zippers for two coinciding H-units (for instance Backerra, 2015 , sections 3.1 and 4.1), then zips $z_{3}$ and $z_{4}$ are empty. Because the sum of actual energy of the four zips is equal to $2 \times V$ and the zips are supposed to have equal potential energies, this implies that for coinciding $\mathrm{H}$-units the actual energy of one zip is equal to $V$. Thus before interacting, the potential energy of one zip also must be equal to $V$. Added to the experience that we never found more than two actual zips in a complete zipper, we suppose that the conversion of potential into actual energy for one zip in general is restricted to this amount, which can be written as:

$$
E\left(z_{i}\right) \leq V
$$

All attributes have to be inserted in general zipper (2) for each quality separately. Only after having reduced all elements according to the considered case, the time zipper will be combined with the space zipper and eventually with the mark zipper. A tilde indicates that the attribute describes a mathematical object. After transformation into a physical space, this indication has to be dropped.

The set of attributes $h_{i}$ of H-unit $H_{i}$ for the quality time $\tilde{t}$ is defined as: 


$$
h_{i}(\tilde{t})=\left\{\widetilde{T}_{i}, \widetilde{F}^{i} \backslash \widetilde{T}_{i}, \tilde{\tau}_{i}, \tilde{f}^{i}\right\}
$$

for the quality space $\mathbf{x}$ as:

$$
h_{i}(\tilde{\mathbf{x}})=\left\{\widetilde{P}_{i}, \tilde{S}^{i} \backslash \widetilde{P}_{i}, \tilde{p}_{i}, \tilde{s}^{i}\right\}
$$

and for the quality mark $\tilde{q}$ as:

$$
h_{i}(\tilde{q})=\left\{\left\{\widetilde{\mathbf{E}}_{i}, \widetilde{Q}_{i}\right\},\left\{\widetilde{\mathbf{B}}^{i}, \widetilde{Q}_{i} \times i\right\},\{\widetilde{\nabla}, 1\},\{\partial / \partial \tilde{t}, i\}\right\} .
$$

Each set of attributes is defined by subsequently two major and two minor attributes, each duo containing one determinate and one indeterminate member. Both major attributes together constitute a closed mathematical system; the same is valid for both minor attributes. The origin of these definitions can be found in previous work (time: see Backerra, 2012, section IV; space: 2012, section III; mark: 2010, section IV and 2015, section 6). Previously the minor attributes of mark were erroneously not included in the set of attributes.

Note that the attributes of time and space are similarly defined, with the only difference that time is defined in one dimension and space in three. It is necessary to split up time and space because by using four-dimensional spacetime, the description of a part of the H-events gets lost. Time is taken as the first quality, because after transformation it does not contain energy, but acts as a label, informing us about the status of the energy of the described H-event.

\subsection{Time Attributes}

Our way of dealing with time is different from classical physics. According to twin physics, time does not run self-evident. If $\mathrm{H}$-units in a closed system have no interaction, which is the case if their major spaces have an empty intersection, then the time in this system stands still. Only if they overlap each other and have interaction, time is existing. Time runs only regularly if the interaction between $\mathrm{H}$-units generates a cyclic phenomenon, providing a clock; in that case, the major point of time will travel along a one-dimensional axis, taking the remaining three attributes of time with it. The past is not incorporated, because it is not observable. In our second publication (Backerra, 2012, section IV) more can be found about the choice of time attributes.

Another very unusual feature of time is, that we do not ascribe energy to time attributes. In twin physics, a time observation acts as a label, attached to an observation, to inform us about the status of the energy, being potential, actual or a combination of both, and if the generated $\mathrm{H}$-event is static or dynamic. An empty time label indicates a potential physical existence, being timeless. A non-empty label indicates the actual existence of an $\mathrm{H}$-event, expressed by exchanged and transformed time attributes. A phenomenon is considered as static if its velocity is zero or constant and it is considered as dynamic if it moves accelerated; this is indicated by the time zipper.

The time attributes are defined as follows. The major determinate attribute is major point of time $\widetilde{T}_{i}$, being the first point of interval $\widetilde{F}^{i}$, which is an arbitrary large span of time. The major indeterminate attribute is future

$$
\widetilde{F}^{i} \backslash \widetilde{T}_{i}=\left\{\tilde{t} \mid \widetilde{T}_{i}<\tilde{t}<\widetilde{T}_{e i}\right\},
$$

in which $\tilde{t}$ is a mathematical point of time. So the future is an interval of time with $\widetilde{T}_{e i}$ at the end. The major time attributes exclude each other, are complementary and constitute together the major time system of $H_{i}$, being $\widetilde{F}^{i}$. The minor indeterminate attribute is the flying time

$$
\tilde{f}^{i}=\left\{\tilde{t} \mid \widetilde{T}_{i} \leq \tilde{t}<\tilde{t}_{i}\right\}
$$

being an extended present. The minor determinate attribute is the flash of time

$$
\tilde{\tau}_{i}=\left\{\tilde{t} \mid \tilde{t}_{i} \leq \tilde{t} \leq \tilde{t}_{i}+d \tilde{t}_{i}\right\},
$$

being an infinitesimal small time interval describing a change. The minor time attributes exclude each other, are complementary and constitute together the minor time system, being $\widetilde{f}^{i} \cup \tilde{\tau}_{i}$.

The time zipper $Z(t)$ is decided by the distance $\widetilde{T}_{i j}$ between $\widetilde{T}_{i}$ and $\widetilde{T}_{j}$ with respect to the flying times and the flashes of time. Previously we distinguished four distinct time cases, but it became apparent that time case 2 had to be split in two separate possibilities and so now we define five time cases. 
Time cases for all interactions: Time case 1 is defined by: $\widetilde{T}_{i}$ and $\widetilde{T}_{j}$ are coinciding, or by: $\widetilde{T}_{i j}=0$. Time case 2 is defined (for $T_{j}>T_{j}$ ) by: $\widetilde{T}_{i}$ exists in the flying time of $H_{j}$ and the flashes of time are partly overlapping, or by: $0<\widetilde{T}_{i j} \leq d \tilde{t}_{i}$. Time case 3 is defined (for $T_{\sim}>T_{j}$ ) by: $\widetilde{T}_{i}$ exists in the flying time of $H_{j}$ and the flashes of time are not overlapping, or by: $d \tilde{t}_{i}<\widetilde{T}_{i j}<\tilde{t}_{i}$. Time case 4 is defined (for $T_{i}>T_{j}$ ) by: $\widetilde{T}_{i}^{j}$ exists in the flash of time of $H_{j}$, or by $\widetilde{t}_{i} \leq \widetilde{T}_{i j} \leq \tilde{t}_{i}+\tilde{d} \tilde{t}_{i}$. Time case 5 is defined by: The overlapping futures do not contain minor attributes, or by: $\widetilde{T}_{i j}>\tilde{t}_{i}+d \tilde{t}_{i}$.

\subsection{Space Attributes}

Our conception of space is different from the classical one. Classical space is infinite; in twin physics, on the contrary, spaces are finite. Classical space has no energy; in twin physics, spaces contain energy. As with time, space exists as a possible result of the interaction of H-units. In classical physics, a space contains an infinite amount of points, each being in principle observable with a certain accuracy; in twin physics one point of an independent space is not observable separately, because the space acts as one organism. In our second publication (Backerra, 2012, section III) you can find a description of the choices of space attributes. They are defined in a three-dimensional mathematical space as follows.

The major determinate attribute is major point of space $\widetilde{P}_{i}$, being a point. The major indeterminate attribute is major space $\widetilde{S}^{i} \backslash \widetilde{P}_{i}$, being a finite sphere with its central point $\widetilde{P}_{i}$ excluded, having a large but not infinite radius $\widetilde{R}$ which might have an astronomic magnitude, the border excluded. The major space attributes exclude each other, are complementary and constitute together the major space system of $H_{i}$, being $\widetilde{S}^{i}$.

The minor indeterminate attribute is minor space $\tilde{\sim}_{\tilde{\sim}}^{i}$, being a small sphere with central point $\widetilde{P}_{i}$, having radius $\tilde{r} \ll<\widetilde{R}$, its border excluded (but $\widetilde{P}_{i}$ included). The minor determinate attribute is pellicle $\widetilde{p}_{i}$, being the border of this minor space, having an infinitely small thickness $d \tilde{r}$. The $\underset{\sim}{\operatorname{sinor}} \underset{\sim}{\text { space attributes exclude each }}$ other, are complementary and constitute together the minor space system, being $\tilde{s} \cup \tilde{p}_{i}$.

The space zipper $Z(\mathbf{x})$ is decided by the distance $\widetilde{P}_{i j}$ of $\widetilde{P}_{i}$ and $\widetilde{P}_{j}$ with respect to the radii of the major spaces $R_{\dot{i}}$ and $R_{\dot{j}}$ and those of the minor spaces $r_{i}$ and $r_{j}$. If the major spaces do not overlap each other at all, so if $\widetilde{P}_{i j} \geq 2 \times R$, then there is no interaction between the H-units.

For a quick comprehension, we advise to use two-dimensional models of H-units, circles cut out of plastic sheets; take the radii for the neutral H-units about five times larger than the marked ones.

Space cases for equally sized H-units (both neutral or both with marking):

For H-units having equal sizes, indicated by $H_{i} * H_{j}$ or by $H_{0 i} * H_{0 j}$ (both marked or both neutral), we define seven space cases, which are given in a geometric as well as an algebraic form.

Case 1 is defined by: $\widetilde{P}_{i}$ and $\widetilde{P}_{j}$ coincide, or by: $\widetilde{P}_{i j}=0$. Case 2 is defined by: $\widetilde{P}_{i}$ and $\underset{\widetilde{P}}{\widetilde{P}} j$ exist inside of each others minor spaces, but they do not coincide, or by: $0<\widetilde{P}_{i j}<\tilde{r}$. Case 3 is defined by: $\widetilde{P}_{i}$ and $\widetilde{P}_{j}$ exist inside each others pellicles, or by: $\tilde{r} \leq \widetilde{P}_{\tilde{P}} \leq \tilde{r}+d \tilde{r}$. Case 4 is defined by: The small spaces are partly overlapping and do not contain $\widetilde{P}_{i}$ and $\widetilde{\sim}_{j}$, or by: $\tilde{r}+d \tilde{r}<\widetilde{P}_{i j}<2 \times \tilde{r}$. Case 5 is defined by: The pellicles touch each other, or by: $2 \times \tilde{r} \leq \widetilde{P}_{i j} \leq 2 \times(\widetilde{r}+d \tilde{r})$. Case 6 is defined by: The major spaces overlap each other such, that the intersection contains all minor attributes, but the pellicles have an empty intersection, or by: $2 \times(\widetilde{r}+d \widetilde{r})<\widetilde{P}_{i j}<\widetilde{R}$. Case 7 is defined by: The major spaces overlap each other partly, but they overlap neither each others major points, nor each others minor attributes, or by: $R+\tilde{r} \leq \widetilde{P}_{i j}<2 \times \widetilde{R}$.

\section{Space cases for mixed sized H-units:}

Interactions of $\mathrm{H}$-units having different sizes (one marked and one neutral) are indicated by

$H_{i} * H_{0 i}$. Marked H-units are smaller than neutral ones; we assume that $R_{0}>R_{i}$ and $r_{0}>r_{i}$. For mixed interactions, only case 7 can be used; below six more cases are defined. The distance of $\widetilde{P}_{i}$ and $\widetilde{P}_{0 i}$ is indicated by $\widetilde{P}_{i 0 i}$. The algebraic description of these cases will not be given if this is superfluous complicated. Mind that previously another numbering of mixed cases is used (Backerra, 2010).

Case 8 is defined by: $\widetilde{P}_{i}$ and $\widetilde{P}_{0 i}$ coincide, or by: $\widetilde{P}_{i}=\widetilde{P}_{0 i}$. Case 9 is defined by: The charged minor space exists inside the neutral one, overlapping the neutral point of space, but the major points do not coincide, or by: $0<P_{i 0 i}<r_{i}$. Case 10 is defined by: The major neutral point exists inside the charged pellicle, or by: $r_{i} \leq P_{i 0 i}<r_{i}+d r$. Case 11 is defined by: The marked minor space exists completely inside the neutral one, not overlapping the neutral major point of space, or by: $r_{i}+d r<P_{i 0 i}<r_{0 i}-\left(r_{i}+d r\right)$. Case 12 is defined by: The marked major point of space exists inside the neutral pellicle, or by: $r_{0 i} \leq P_{i 0 i} \leq r_{0 i}+d r$. Case 13 is defined by: the major points of space exist in each others major spaces, but their minor spaces and pellicles have empty intersections, or by: 
$r_{i}+r_{0 i}+2 \times d r<P_{i 0 i}<R_{i}$. Case 14 is defined by: The marked minor space exists completely inside the neutral major space, but the marked major space is not overlapping the neutral major point of space, or by: $R_{i} \leq P_{i 0 i} \leq R_{0 i}-r_{i}$.

\subsection{Mark Attributes}

Mark attributes are defined in a complementary way, like we did with space and time attributes, and such that an entire $\mathrm{H}$-unit is distinguished from another one by having a positive or negative charge. If an $\mathrm{H}$-unit is not marked, it is called neutral. More about the quality mark can be found in our first and fourth paper (Backerra, 2010, the introduction of section IV, and 2015, section 6). The mark attributes are as follows defined in mathematical time and three-dimensional mathematical space.

First we consider the major mark attributes. In the major determinate mark set $\left\{\widetilde{\mathbf{E}}_{i}, \widetilde{Q}_{i}\right\}$, electric field $\widetilde{\mathbf{E}}_{i}(\tilde{t})$ is defined as a 3-dimensional, radial oriented, time dependent vector field with $\underset{\widetilde{\mathbf{E}}}{\widetilde{\mathbf{E}}_{i}}\left(\widetilde{P}_{i}\right)=\mathbf{0}$, and its source is real charge $\widetilde{Q}_{i}$, defined as a real number. In major indeterminate mark set $\left\{\widetilde{\mathbf{B}}^{i}, \widetilde{Q}_{i} \times i\right\}$, magnetic field $\widetilde{\mathbf{B}}^{i}(\tilde{t})$. is a 3-dimensional, time dependent vector field with $\widetilde{\mathbf{B}}^{i}\left(\widetilde{P}_{i}\right)=\mathbf{0}$, and its source is imaginary charge $\widetilde{Q}_{i} \times i$ (with $i=\sqrt{-1}$ ), defined as an imaginary number. The magnetic field has by definition one vector in each point, having a direction tangent to a spherical surface with $\widetilde{P}_{i}$ as the central point. Adjacent vectors may point in an infinite variety of directions and there is no information about the absolute value of each vector. To create an $\underset{\widetilde{\mathbf{B}}}{\text { absolute }} \widetilde{\mathbf{B}}^{j}$ indeterminate magnetic field, the principle of uniqueness is added to the definition of $\widetilde{\mathbf{B}}^{i}$, saying that $\widetilde{\mathbf{B}}^{i} \neq \widetilde{\mathbf{B}}^{j}$ for any two H-units $H_{i}$ and $H_{j}$.

Secondly we define the minor mark attributes. In the minor determinate mark set $\{\widetilde{\nabla}, 1\}, \widetilde{\nabla}$ is defined as the nabla operator $(\partial / \partial \tilde{x}, \partial / \partial \tilde{y}, \partial / \partial \tilde{z})$ and the number 1 is the unity of real numbers. In the minor indeterminate mark set $\{\partial / \partial \tilde{t}, i\}, \partial / \partial \tilde{t}$ is defined as the mathematical time derivative and $i$ is defined by $i=\sqrt{-1}$. Mark zipper $Z(q)$ is decided by the charges $\widetilde{Q}_{i}$ and $\widetilde{Q}_{j}$.

\section{Mark cases for all interactions:}

Mark case 1 is defined by $\widetilde{Q}_{i}=\widetilde{Q}_{j}$. Mark case 2 is defined by $\widetilde{Q}_{i}=-\widetilde{Q}_{j}$. Mark case 3 is defined by $\widetilde{Q}_{0 i}=0$. In the third case, a marked H-unit $H_{i}$ interacts with a neutral one $H_{0 i}^{j}$; mind that the size of the marked $\mathrm{H}$-unit is smaller than that of the neutral one.

Previously we called $\widetilde{\mathbf{E}}_{i}(\tilde{t})$ and $\widetilde{\mathbf{B}}^{i}(\tilde{t})$ 'determinate and indeterminate vector fields' and $\widetilde{Q}_{i}$ and $\widetilde{Q}_{i} \times i$ 'real and imaginary numbers'; only after transformation we called them 'electric and magnetic fields' and 'charges'. Later we switched over to the same indication for both conditions, remembering that a tilde indicates a mathematical existence, and the absence of a tilde a physical existence; they may occur mixed in cases that we do not know yet all transformations.

\subsection{Operators}

To obtain rules for interacting major and minor objects, we will return to the extended Heisenberg's uncertainty principle, saying that each 'certain observation' contains a small influence of 'uncertainty', and the other way round, each 'uncertain observation' contains a small influence of 'certainty'. The operator 'joining' is defined such that these conditions are met, and in a similar way for time and space. Mind that the difference in dimensions of time and space in some cases may result in zippers which are not at all similar.

Joining of time attributes, if major points of time $T$ are involved, is defined as:

$$
\begin{aligned}
& T_{i} \bowtie f^{j}=f^{j} \text { if } T_{i} \in f^{j} ; T_{i} \triangleright \triangleleft f^{j}=\varnothing \text { if } T_{i} \notin f^{j} ; \\
& T_{i} \triangleright \tau_{j}=\tau_{j} \text { if } T_{i} \in \tau_{j} ; T_{i} \triangleright \triangleleft \tau_{j}=\varnothing \text { if } T_{i} \notin \tau_{j}
\end{aligned}
$$

and if major futures $F$ are involved, as:

$$
\begin{aligned}
& F^{i} \triangleright \triangleleft \tau_{j}=\tau_{j} \text { if } \tau_{j} \subset F^{i} ; F^{i} \triangleright \triangleleft \tau_{j}=\varnothing \text { if } \tau_{j} \not \subset F^{i} ; \\
& F^{i} \triangleright f^{j}=f^{j} \text { if } f^{j} \subset F^{i} ; F^{i} \triangleright f^{j}=\varnothing \text { if } f^{j} \not \subset F^{i}
\end{aligned}
$$

in which $\varnothing$ indicates an empty set.

Linking of joined pairs of time is defined as taking their intersection.

Joining of space attributes, if major points of space $P$ are involved, is defined as: 


$$
\begin{aligned}
& P_{i} \bowtie s^{j}=s^{j} \text { if } P_{i} \in s^{j} ; P_{i} \bowtie s^{j}=\varnothing \text { if } P_{i} \notin s^{j} ; \\
& P_{i} \triangleright p_{j}=p_{j} \text { if } P_{i} \in p_{j} ; P_{i} \triangleright \triangleleft p_{j}=\varnothing \text { if } P_{i} \notin p_{j} .
\end{aligned}
$$

and if major spaces $S$ are involved:

$$
\begin{aligned}
& S_{\triangleright \triangleleft}^{i} p_{j}=p_{j} \text { if } p_{j} \subset S^{i} ; S^{i} \bowtie p_{j}=\varnothing \text { if } p_{j} \not \subset S^{i} ; \\
& S^{i} \bowtie S^{j}=s^{j} \text { if } s^{j} \subset F^{i} ; S^{i} \bowtie S^{j}=\varnothing \text { if } s^{j} \not \subset S^{i} .
\end{aligned}
$$

Linking of joined pairs of space is defined as taking their intersection.

Joining of mark attributes is defined for fields and charges separately.

For fields, joining is defined as:

$$
\widetilde{\mathbf{A}} \bowtie \widetilde{\nabla}=\widetilde{\nabla} \times \widetilde{\mathbf{A}} \text { and } \quad \widetilde{\mathbf{A}} \bowtie \partial / \partial \tilde{t}=\partial \widetilde{\mathbf{A}} / \partial \tilde{t},
$$

in which $\widetilde{\nabla} \times \widetilde{\mathbf{A}}$ is a vector-product.

For charges, joining is defined as multiplication of major attributes with minor attributes 1 and i, subsequently. This results in the possible pairs of mark attributes $\widetilde{Q}_{i}, \widetilde{Q}_{i} \times i$ and $-\widetilde{Q}_{i}$.

Linking of joined pairs of mark attributes is as well defined for fields and charges separately.

For fields, linking is defined as vector addition of the joined pairs; it is important to carry out this operation after joining. According to the definition of linking, two equal vector fields link to a single vector field, so if $\widetilde{\mathbf{E}}_{i}=\widetilde{\mathbf{E}}_{j}$ than $\widetilde{\mathbf{E}}_{i} \propto \widetilde{\mathbf{E}}_{j}=\widetilde{\mathbf{E}}_{i}$. Note that electric fields are only equal in case they have two equal charges and two coinciding major points of space. Magnetic fields cannot be equal, because of the principle of uniqueness.

For charges, linking is defined as numerical addition. Two equal charges link to one single charge, so if $\widetilde{Q}_{i}=\widetilde{Q}_{j}$ than $\widetilde{Q}_{i} \propto \widetilde{Q}_{j}=\widetilde{Q}_{i}$.

The transformation of a mathematical element $\widetilde{X}$ into a physical item $X$ is written as $[\widetilde{X}]=X$; by carrying out this transformation, tildes above attributes disappear. The transformations of time elements of a zip are defined by

$$
\left[\widetilde{T}_{i}\right]=T_{i} ;\left[\widetilde{F}^{i} \backslash \widetilde{T}_{i}\right]=F^{i} \backslash T_{i} ;\left[\tilde{\tau}_{i}\right]=\tau_{i} ;\left[\tilde{f}^{i}\right]=f^{i},
$$

\section{Transformed time elements do not contain energy.}

The transformations of space elements of a zip are defined by

$$
[\widetilde{P}]=P ;[\tilde{S} \backslash \widetilde{P}]=S \backslash P ;\left[\tilde{s}^{i}\right]=s^{i} ;\left[\tilde{p}_{i}\right]=\pi\left(p_{i}\right) ;\left[\tilde{p}_{i} \cap \tilde{p}_{j}\right]=\pi\left(p_{i} \cap p_{j}\right) .
$$

The last two objects are minor spherical spheres, occupying the full width of the pellicle.

A new definition is introduced for the transformation of the small-scale geometric element $s^{i} \cap p_{j}$ in zip $z_{3}$ (and mirrored in $z_{4}$ ):

$$
\left[\tilde{s}^{i} \cap \tilde{p}_{j}\right]=e_{s^{i} \cap p_{j}},
$$

in which $e$ is a tiny sphere. This definition affiliates better with the two previous definitions in which pellicles are involved and spherical minor particles are generated. This geometric object will be considered further in section 2.5. Transformed space elements contain energy proportional to the magnitude of the space, and so a point of space contains no energy.

The transformations of mark elements of a zip are defined by:

$$
\begin{aligned}
& {\left[\widetilde{\mathbf{E}}_{i}\right]=\mathbf{E}_{i} ;\left[\widetilde{\mathbf{B}}^{i}\right]=\mathbf{B}^{i} ;\left[\widetilde{\nabla} \times \widetilde{\mathbf{E}}_{i}\right]=\nabla \times \mathbf{E}_{i} ;\left[\widetilde{\nabla} \times \widetilde{\mathbf{B}}^{i}\right]=\nabla \times \mathbf{B}^{i} ;} \\
& {\left[\widetilde{Q}_{i}\right]=Q_{i} ;\left[\widetilde{Q}_{i} \times i\right]=0 ;\left[-\widetilde{Q}_{i}\right]=-Q_{i}}
\end{aligned}
$$

Because the electric field is decided by its charge, and because three distinct transformations of charge exist, we distinguish three types of H-units: positively marked, negatively marked, and neutral. In general marked $\mathrm{H}$-units 
are written as $H_{i}$ and neutral ones as $H_{0}$.

All transformed mark elements add actual energy to geometric items, on the understanding that the mathematical charges and infinite vector fields after transformation are restricted to the described time and space elements of the zip. The actual energy of charge $Q_{i}$ is defined as a constant.

After having transformed the zipper completely, and thus having removed all square brackets, the potential energy of each zip is partly or fully converted into actual energy. As we saw in equation (5), the actual energy of a zip (so the actual energy of an H-event) cannot exceed the unity of potential energy, which is $V$. The final interpretation in physical reality of zip $z_{i}(t, \mathbf{x}, q)$, is called the appearance $A_{i}\left(z_{i}\right)$, describing an H-event, which is a phenomenon having actual energy. The set of appearances of the zipper is defined as a set containing one interpretation of each zip, which can be written as:

$$
A(Z(t, \mathbf{x}, q))=\left\{A_{1}(t, \mathbf{x}, q), A_{2}(t, \mathbf{x}, q), A_{3}(t, \mathbf{x}, q), A_{4}(t, \mathbf{x}, q)\right\} .
$$

As far as we have found, for each case maximum two appearances of a zipper are non-empty.

If the transformed zip contains actual energy but the appearance is not observable, the appearance will be written between round brackets, so as $\left(A_{i}(t, \mathbf{x}, q)\right)$. If desirable, the qualities $t, \mathbf{x}$ and $q$ may be dropped in the formulation and replaced by more relevant indications.

\subsection{Defining Appearances of Actual Energetic Items}

It is important to understand the difference between the notion of energy by common consent and that in twin physics. Because both mass and space are generated by interacting H-units, both are considered as energetic spatial objects, called energetic space. The difference between mass and space is supposed to be only in the concentration of energy. An actual object with a high energetic density is called a compact space, an actual object with low energetic density is called an extended space. For convenience 'compact space' and 'extended or free space' may be expressed in the classical terms 'mass' and 'space'. We suppose that these two energy densities are constants. Moreover, we suppose that the energy is homogeneously distributed over the occupied volume.

The quality time carries no energy. Time is a label of energy, informing us about the status of a physical item, existing potentially or actual, in the present or in the future, in a static or a dynamic way. Note that the past is not represented.

By marking an H-unit, it is supplied with potential mark energy. Because the potential energy of an H-unit is constant, this implies that by marking, the potential space energy will be reduced and thus the sizes of the space attributes are reduced. This implies that the total energy density of a marked energetic space is higher than that of a neutral energetic space. Supposing that for a marked H-unit the proportion of potential space and mark energy is a constant, and that the two possible densities of mark energy also are constants, we obtain four distinct energy densities. The highest one is related to charged mass, followed by neutral mass, marked space, and neutral space.

Below we will list the chosen names for appearances of actual energetic objects. They are only related to the geometric appearances, without considering the quality 'mark'.

First we consider extended spaces. The appearance of (a part of) a major space is called major extended space $\Theta$. The appearance of (a part of) a minor space is called minor extended space $\theta$. A space without an accompanying particle in another zip, is called a free space; its two types are major free space $\Theta^{0}$ and minor free space $\Theta^{0}$.

Second we consider compact spaces, which may appear as major or minor mass-carrying particles or as a point particle. The appearance of $s^{i} \cap s^{j}$ or $S^{i}$ is called a major particle $\sigma$; it may be identified with a proton or a neutron. The appearance of $p_{i} \cap p_{j}$ is called a minor particle $\pi$, being a relatively small sphere existing inside the intersecting pellicles, such that the width is maximum used, having no charge (because the second zip, in which it appears, cannot describe charge); its actual energy is equal to the potential energy of the complete mathematical object $p_{i} \cap p_{j}$. It may be identified with a gluon or a neutrino. The appearance of $s^{i} \cap p_{j}$ is called a dot particle $\delta$, being a relatively very small sphere, possibly charged (because the third or fourth zip, in which it appears, may describe charge), existing inside the indicated geometric object, having an actual energy equal to the potential energy of the complete mathematical object; it may be identified with an electron (previously the transformation to a small sphere was not yet defined, as we supposed that the disc-like form could be actual). 
Third we consider items having neither an extended, nor a compact space. The appearance of point of space $P_{i}$ is called a point particle $\Pi$; this carries no spatial energy, but it might carry mark energy in the form of a charge or an electromagnetic field; it may be identified with a photon.

\subsection{Reducing the Zipper to Identify H-events}

With the theoretical tools as given above, we are ready to deduce time, space and mark zippers in specific cases, based upon the general zipper (2). First we carry out the operation joining, and second linking (see section 2.4). After that, the zippers will be combined into one time-space-mark zipper. Each non-zero zip of the zipper is a set, containing two elements; the first is a stripped chain and the second is a chain of exchanged attributes. Each zip has to be transformed into a physical space. For some elements this is obvious; however, often it is not clear how to transform. Below we give some indications how to combine the large- and small-scale elements of each zip into a phenomenon.

The most easy indication is that, if both corresponding elements of a zip contain empty time elements, no potential energy can be converted into actual energy. The same is valid for corresponding empty space elements. In that case, the zip will be replaced by an empty set. If only one time element is empty, the belonging geometric element might exist independent of time.

Corresponding large- and small-scale elements have to be compatible for each quality. If, for instance, a large-scale geometric point occurs in combination with a small-scale geometric object, then we require that the point is an element of the object. If this is not possible, the two elements are not compatible and the zip will be replaced by an empty set. This requirement can be compared with an every-day zipper, having a series of left and right "teeth" which closes properly only by bringing them in the right contact with each other.

If a large-scale element is empty in all three qualities, the small-scale element still may be observable and so an $\mathrm{H}$-event might exists. On the contrary, if the small-scale element is empty in all three qualities, the observation is incompatible with the extended Heisenberg's principle, implying that a large scale attribute cannot be observed without a small scale addition. In that case there might be an H-event, so potential energy might be converted into actual energy, but the resulting H-event is not observable. Previously we replaced the zip by an empty set in that case, but in describing the photon, it appeared to be useful to describe unobservable actual items as well; then the resulting physical item will be placed between round brackets.

After these first checks, we are ready for the fine-tuning of interpretations. The complementary starting-point of twin theory is reflected in each zip in a different way, so we have to consider each zip separately.

2.6.1 The first zip mainly describes the determinate aspects of nature, so concentrated items, which is expressed by large-scale element $D_{i} \propto D_{j}$. The combination of the large- and small-scale element may describe a mass-carrying particle, geometric characterized by the small-scale element and localized by the large-scale element. Thus the small-scale element softens the extreme determinate character of the large-scale element.

2.6.2 The second zip mainly describes the indeterminate aspect of nature, so divergent items like space, which is expressed by large-scale element $U^{i} \propto U^{j}$. The large-scale element of the second zip is non-empty for each interaction, in whatever case. Previously we supposed that space was generated in all cases, the small-scale element adding specific details. This was an erroneous reflex, originating in having considered space in the classically way, so as 'always and everywhere existing'. If in each interaction actual space would be generated, having a constant energy density, then a larger number of interacting H-units, generating many overlapping spaces, would cause an energetic problem, because space is supposed to have a constant energy density. We discovered that, if the small-scale geometry describes a minor particle, then the large-scale space has to be reduced to the spatial track of this particle. Then the small-scale element softens the extreme indeterminate character of the large-scale space. Below we consider three distinct possibilities for the second zip, the first two of them depending on the first zip.

If zip $z_{1}$ describes a heavy mass-carrying particle $\sigma$ (see section 2.5 ), then $\left[\tilde{p}_{i} \cap \tilde{p}_{j}\right]$ in $z_{2}$ is defined as a light-weight minor particle $\pi$ appearing in the intersection of pellicles around the mass. Because there is no boundary between them, $\sigma$ and $\pi$ may be considered together as one single H-event, in which $\pi$ is identified with a gluon, providing the spin of the particle (see Backerra, 2012, VII.E). The large-scale element of $z_{2}$, being $\tilde{S}^{i} \cap \tilde{S}^{j}$, is reduced to the track of the gluon.

If zip $z_{1}$ is empty, then the transformation of $\left[\tilde{p}_{i} \cap \tilde{p}_{j}\right]$ in $z_{2}$ can be defined as a lightweight minor particle $\pi$, existing independently of another particle. This particle is identified with a neutrino, its radius depending on the type of involved pellicles. Thus the large-scale element of $z_{2}$, being $\widetilde{S}^{i} \cap \widetilde{S}^{j}$, is reduced to the track of the neutrino. 
If the small-scale space element of $z_{2}$ is empty, then the generated space $\tilde{S}^{i} \cap \tilde{S}^{j}$, containing actual energy, is unobservable and the appearance (see section 2.5) will be written between round brackets as $(\Theta)$. If two neutral H-units are interacting, then the space is written as $S^{0 i} \cap S^{0 j}$ and the appearance is an unobservable major extended space $\left(\Theta^{0}\right)$; if a small-scale element of time is indicated ( $\tau_{0 i}$ or $\left.\tau_{0 i} \cap \tau_{0 j}\right)$ without a small-scale geometry, then this element will be assigned to the major space, so the space is moving. If two marked $\mathrm{H}$-units are interacting, than the generated space is smaller and magnetized; the appearance is $\left(\Theta^{\mathbf{B}}\right)$, being an actual, unobservable, magnetized extended space.

2.6.3 The third and fourth zip are symmetrical with respect to the indices. Mind that the resulting H-events in general are not symmetrical, which will become clear by considering mixed cases (the interaction of a marked and a neutral H-unit). These two zips mainly describe the exchange between determinate and indeterminate aspects in nature, like in electromagnetic waves, which is expressed by their large-scale elements $D_{i} \propto U^{j}$ and $D_{j} \propto U^{i}$. If one time or one geometric element is empty, the mark element exists independent of time or space and will be ascribed to the corresponding element of the same zip.

\subsection{Time Zippers, Space Zippers and Mark Zippers}

The zippers for separate qualities are derived by inserting the sets of attributes (6), (7) and (8) in the general zipper (2) and adapt them to the cases in consideration (sections 2.1, 2.2 and 2.3). A tilde above a symbol says that it is a mathematical symbol; square brackets around an element say that the element has to be transformed to a real physical space, possibly even when the attributes are already transformed. Different as in sections 2.1 and 2.2 , the cases are not described in a geometric or functional algebraic way any more, but in set algebra according to Kahn (1967).

For time zippers, all elements are transformed, and so all square brackets and tildes are removed. All space attributes are, as a first step, transformed to a physical space, but if an element involves pellicles, it is still written with square brackets, because it may be a non-singular object; only after having considered the remaining zips, the final appearance can be obtained. For mark zippers, the operations joining, linking and transforming are carried out as far as possible, but in general they can be carried out only after the time and space parts are completely transformed.

\section{Time zippers:}

Cases 2 through 5 are defined for $T_{i}>T_{j}$; for $T_{i}<T_{j}$ the zipper is found by interchanging indices $i$ by $j$, and the same for zips $z_{3}$ and $z_{4}$. All elements are transformed to physical time items, so no tildes have to be written any more.

Case 1 (so $\left.T_{i} \cap T_{j}=\varnothing\right)$ :

$$
Z(t)=\left\{\left\{T_{i}, f^{i}\right\},\left\{F^{i} \backslash T_{i}, \tau_{i}\right\}, \varnothing, \varnothing\right\}
$$

Case 2 (so $T_{i} \in f^{j}$ and $\tau_{i} \cap \tau_{j} \neq \varnothing$ ):

$$
Z(t)=\left\{\varnothing,\left\{F^{i} \backslash T_{i} \cap F^{j}, \tau_{i} \cap \tau_{j}\right\},\left\{T_{i}, \varnothing\right\}, \varnothing\right\} .
$$

Case 3 (so $T_{i} \in f^{j}$ and $\tau_{i} \cap \tau_{j}=\varnothing$ ):

$$
Z(t)=\left\{\varnothing,\left\{F^{i} \backslash T_{i} \cap F^{j}, \varnothing\right\},\left\{T_{i}, \varnothing\right\}, \varnothing\right\} .
$$

Case 4 (so $T_{i} \in \tau_{j}$ ):

$$
Z(t)=\left\{\varnothing,\left\{F^{i} \backslash T_{i} \cap F^{j}, \varnothing\right\},\left\{T_{i}, f^{i} \cap \tau_{j}\right\}, \varnothing\right\} .
$$

Case 5 (so $T_{i} \in F^{j}$ and $\left.F^{i} \cap \tau_{j}=\varnothing\right)$ :

$$
Z(t)=\left\{\varnothing,\left\{F^{i} \backslash T_{i} \cap F^{j}, \varnothing\right\},\left\{T_{i}, \varnothing\right\}, \varnothing\right\} .
$$

Space zippers:

First the zippers for equally sized H-units are given; after that, the zippers for mixed H-units. All attributes are transformed to physical space items, so no tildes any more. However, if an element contains a pellicle, it is still written between square brackets because the transformation cannot yet be completed. 
Case 1 for H-units of equal size, with $P_{i} \cap P_{j}=P_{i}$ :

$$
Z(\mathbf{x})=\left\{\left\{P_{i}, s^{i}\right\},\left[S^{i} \backslash P_{i}, p_{i}\right], \varnothing, \varnothing\right\} .
$$

Case 2 for H-units of equal size, with $P_{i} \in s^{i} \cap s^{j}$ and $P_{i} \cap P_{j}=\varnothing$ :

$$
Z(\mathbf{x})=\left\{\left\{\varnothing, s^{i} \cap s^{j}\right\},\left[S^{i} \backslash P_{i} \cap S^{j} \backslash P_{j}, p_{i} \cap p_{j}\right],\left\{P_{i}, \varnothing\right\},\left\{P_{j}, \varnothing\right\}\right\} .
$$

(Note that $z_{1}(\mathbf{x})$ contains $s^{i} \cap s^{j}$, whilst $z_{1}(t)=\varnothing$ because time is one-dimensional).

Case 3 for H-units of equal size, with $P_{i} \in p_{j}$ and thus $P_{j} \in p_{i}$ :

$$
Z(\mathbf{x})=\left\{\varnothing,\left[S^{i} \backslash P_{i} \cap S^{j} \backslash P_{j}, p_{i} \cap p_{j}\right],\left\{P_{i}, s^{i} \cap p_{j}\right\},\left\{P_{j}, s^{j} \cap p_{i}\right\}\right\} .
$$

Case 4 for $\mathrm{H}$-units of equal size, with $s^{i} \cap s^{j} \neq \varnothing$ and $P_{i} \notin s^{i} \cap s^{j}$ :

$$
Z(\mathbf{x})=\left\{\varnothing,\left[S^{i} \backslash P_{i} \cap S^{j} \backslash P_{j}, p_{i} \cap p_{j}\right],\left\{P_{i}, \varnothing\right\},\left\{P_{j}, \varnothing\right\}\right\} .
$$

Case 5 for H-units of equal size, with $p_{i} \cap p_{j}=p_{i j}$ in which $p_{i j}$ is a singular object:

$$
Z(\mathbf{x})=\left\{\varnothing,\left[S^{i} \backslash P_{i} \cap S^{j} \backslash P_{j}, p_{i j}\right],\left\{P_{i}, \varnothing\right\},\left\{P_{j}, \varnothing\right\}\right\} .
$$

Case 6 for H-units of equal size, with $P_{i} \in S^{i} \cap S^{j}$ and $P_{j} \in S^{i} \cap S^{j}$, but $p_{i} \cap p_{j}=\varnothing$ :

$$
Z(\mathbf{x})=\left\{\varnothing,\left\{S^{i} \backslash P_{i} \cap S^{j} \backslash P_{j}, \varnothing\right\},\left\{P_{i}, \varnothing\right\},\left\{P_{j}, \varnothing\right\}\right\} .
$$

Case 7 for two H-units in general, so $S^{i} \cap S^{j} \neq \varnothing$, with $p_{i} \notin S^{j}$ and $p_{j} \notin S^{i}$ :

$$
Z(\mathbf{x})=\left\{\varnothing,\left\{S^{i} \cap S^{j}, \varnothing\right\}, \varnothing, \varnothing\right\} .
$$

Case 8 for H-units of different size, with $P_{i} \cap P_{0 i}=P_{i}$ :

$$
Z(\mathbf{x})=\left\{\left\{P_{i}, s^{i}\right\},\left\{S^{i} \backslash P_{i}, \varnothing\right\}, \varnothing, \varnothing\right\} .
$$

Case 9 for H-units of different size, with $P_{0 i} \in s^{i}$ and $P_{i} \cap P_{0 i}=\varnothing$ :

$$
Z(\mathbf{x})=\left\{\left\{\varnothing, s^{i}\right\},\left\{S^{i} \backslash P_{i}, \varnothing\right\},\left\{P_{i}, \varnothing\right\},\left\{P_{0 i}, \varnothing\right\}\right\} .
$$

Case 10 for H-units of different size, with $P_{0 i} \in p_{i}$ :

$$
Z(\mathbf{x})=\left\{\varnothing,\left\{S^{i} \backslash P_{i}, \varnothing\right\},\left\{P_{i}, \varnothing\right\},\left[P_{0 i}, p_{i}\right]\right\}
$$

Case 11 for H-units of different size, with $s^{i} \subset s^{0 i}$ and $P_{0 i} \notin s$ :

$$
Z(\mathbf{x})=\left\{\varnothing,\left\{S^{i} \backslash P_{i}, \varnothing\right\},\left\{P_{i}, \varnothing\right\},\left\{P_{0 i}, \varnothing\right\}\right\} .
$$

Case 12 for H-units of different size, with $P_{i} \in p_{0 i}$ :

$$
Z(\mathbf{x})=\left\{\varnothing,\left[S^{i} \backslash P_{i} \cap S^{0 i} \backslash P_{0 i}, p_{i} \cap p_{0 i}\right],\left\{P_{i}, s^{i} \cap p_{0 i}\right\},\left\{P_{0 i}, \varnothing\right\}\right\} .
$$

Case 13 for H-units of different size, with $P_{i} \cap S^{0 i} \neq \varnothing$ and $P_{0 i} \cap S^{i} \neq \varnothing$, but $p_{i} \cap p_{0 i}=\varnothing$ :

$$
Z(\mathbf{x})=\left\{\varnothing,\left[S^{i} \backslash P_{i} \cap S^{0 i} \backslash P_{0 i}, \varnothing\right],\left\{P_{i}, \varnothing\right\},\left\{P_{0 i}, \varnothing\right\}\right\} .
$$

Case 14 for H-units of different size, with $S^{i} \subset S^{0 i}$ and $P_{0 i} \notin S^{i}$ :

$$
Z(\mathbf{x})=\left\{\varnothing,\left\{S^{i} \backslash P_{i} \cap S^{0 i} \backslash P_{0 i}, \varnothing\right\},\left\{P_{i}, \varnothing\right\}, \varnothing\right\} .
$$


Mark zippers:

The mark zipper for two marked H-units $H_{i}$ and $H_{j}$ in case $1\left(Q_{i}=Q_{j}\right)$ and in case $2\left(Q_{i}=-Q_{j}\right)$ is:

$$
Z(q)=\left\{\begin{array}{l}
{\left[\left\{\widetilde{Q}_{i} \propto \widetilde{Q}_{j}, \widetilde{\mathbf{E}}_{i} \propto \widetilde{\mathbf{E}}_{j}\right\}, \partial \widetilde{\mathbf{E}}_{i} / \partial \tilde{t} \propto \partial \widetilde{\mathbf{E}}_{j} / \partial \tilde{t}\right]} \\
{\left[\left\{\left\{0, \mathbf{B}^{i}+\mathbf{B}^{j}\right\}\right\},\left\{\widetilde{\nabla} \times \mathbf{B}^{i} \propto \widetilde{\nabla} \times \widetilde{\mathbf{B}}^{j}\right\}\right]} \\
{\left[\left\{\widetilde{Q}_{i}, \widetilde{\mathbf{E}}_{i} \propto \widetilde{\mathbf{B}}^{j}\right\}, \partial \widetilde{\mathbf{E}}_{i} / \partial \tilde{t} \propto \widetilde{\nabla} \times \widetilde{\mathbf{B}}^{j} \propto \widetilde{\nabla} \times \widetilde{\mathbf{E}}_{i} \propto \partial \widetilde{\mathbf{B}}^{j} / \partial \tilde{t}\right]} \\
{\left[\left\{\widetilde{Q}_{j}, \widetilde{\mathbf{E}}_{j} \propto \widetilde{\mathbf{B}}^{i}\right\}, \partial \widetilde{\mathbf{E}}_{j} / \partial \tilde{t} \propto \widetilde{\nabla} \times \widetilde{\mathbf{B}}^{i} \propto \widetilde{\nabla} \times \widetilde{\mathbf{E}}_{j} \propto \partial \widetilde{\mathbf{B}}^{i} / \partial \tilde{t}\right]}
\end{array}\right\} .
$$

Because the fields are time and space dependent, the mark zipper can only be transformed further after combining with the time and the space zippers. In case 1: $\widetilde{Q}_{i} \propto \widetilde{Q}_{j}=\widetilde{Q}_{i}$. In case 2: $\widetilde{Q}_{i} \propto \widetilde{Q}_{j}=0$.

In case $3\left(\widetilde{Q}_{0 i}=0\right)$, the non-existing fields and charges of $H_{0 i}$ in (41) are dropped and the zipper is:

$$
Z(q)=\left\{\begin{array}{l}
\left\{\left\{Q_{i}, \mathbf{E}_{i}\right\}, \partial \mathbf{E}_{i} / \partial t\right\} \\
\left\{\left\{0, \mathbf{B}^{i}\right\}, \nabla \times \mathbf{B}^{i}\right\} \\
\left\{\left\{Q_{i}, \mathbf{E}_{i}\right\},\left\{\partial \mathbf{E}_{i} / \partial t+\nabla \times \mathbf{E}_{i}\right\}\right\} \\
\left\{\left\{0, \mathbf{B}^{i}\right\},\left\{\nabla \times \mathbf{B}^{i}+\partial \mathbf{B}^{i} / \partial t\right\}\right\}
\end{array}\right\} .
$$

The limiting conditions (for the derivation: see Backerra, 2015), are in general:

$$
\widetilde{\nabla} \cdot \widetilde{\mathbf{E}}_{i}=\widetilde{Q}_{i} ; \widetilde{\nabla} \cdot \widetilde{\mathbf{B}}^{i}=0 ; \partial \widetilde{\mathbf{E}}_{i} / \partial \tilde{t}=\widetilde{\nabla} \times \widetilde{\mathbf{B}}^{i} ; \partial \widetilde{\mathbf{B}}^{i} / \partial \tilde{t}=-\widetilde{\nabla} \times \widetilde{\mathbf{E}}_{i}
$$

and similar for transformed fields (without tildes). Note that two identically marked H-units are in general not identical in geometrical sense. Bear in mind that identical magnetic fields cannot exist, due to the principle of uniqueness.

\section{Gravity and Electricity}

In our previous paper about gravity (Backerra, 2014), we considered only zippers of the first order, in the erroneous conviction that zips of the second order could not be identified with gravity. Because there turned out to be no difference between the results of first and second order zippers, we will consider this subject now with zippers of only the second order.

Essential for the existence of gravity is, that spatial attributes of neutral H-units are larger than those of marked H-units, because they do not have to spend potential energy to the quality mark. For convenience we assume that $S^{0 i}>>S^{i}$ and $s^{0 i}>>s^{i}$, which means that the difference in size is large enough for our considerations (and the same for index $j$ ). Like in the previous paper, we will consider two charged particles $\sigma_{i}$ and $\sigma_{j}$, each generated by the interaction of one marked and one neutral $\mathrm{H}$-unit. The marked $\mathrm{H}$-units are indicated by $H_{i}$ and $H_{j}$, the neutral ones by $H_{0 i}$ and $H_{0 j}$. There are six combinations of two H-units out of four, so in principle six interactions. In this paper, we consider time cases as distinct ways of labeling separate interactions and so these six interactions, participating in the gravitational process, may be described by distinct time cases. In this paper we do not consider the possible physical meanings of differences in time cases, as we want to consider only the energetic process and time does not contain energy.

\subsection{Generation of Two Stable, Charged Particles}

Previously we did not pay much attention to the exact description of the particles, involved in the gravitational process. Only now we found a mistake (Backerra, 2014, section IV.A, equation (55)), describing them in time case 2, but then the relevant element is empty; these particles can only be described in time case 1 . However, it makes no difference for the considerations about gravity. This time we will go more into the details of the particles and deduce the zippers properly in time case 1.

Two stable, charged particles, existing isolated from each other, are described by the two interactions $H_{i} * H_{0 i}$ and $H_{j} * H_{0 j}$. The zipper is considered in time case 1 , space case 8 and mark case 3 , which means that we 
have mixed H-units with $T_{i} \cap T_{0 i}=T_{i}, P_{i} \cap P_{0 i}=P_{i}$ and $Q_{0 i}=0$. Then the zipper for $H_{i}^{*} H_{0 i}$ can be written as (see (22), (34) and (42):

$$
Z(t, \mathbf{x}, q)=\left\{z_{1}, z_{2}\right\}=\left\{\begin{array}{l}
{\left[\left\{T_{i}, P_{i},\left\{Q_{i}, \mathbf{E}_{i}\right\}\right\},\left\{f^{i}, s^{i}, \partial \mathbf{E}_{i} / \partial t\right\}\right]} \\
{\left[\left\{F^{i} \backslash T_{i} \cap F^{j} \backslash T_{j}, S^{i} \backslash P_{i}, \mathbf{B}^{i}\right\},\left\{\tau_{i}, \varnothing, \nabla \times \mathbf{B}^{i}\right\}\right]}
\end{array}\right\} .
$$

The mark elements can not completely be transformed yet and so the square brackets are still present.

In the first zip $z_{1}$, the small-scale mark element $\partial \mathbf{E}_{i} / \partial t$ must be a constant field of derivatives, because time element $f^{i}$ cannot describe a change, so it is called $\mathbf{C}$. This field exists inside $s^{i}$, so the particle moves with a constant velocity with respect to another particle, but because it is an isolated particle, the velocity is zero. The small-scale mark element of the second zip $z_{2}$ is $\nabla \times \mathbf{B}^{i}=\partial \mathbf{E}_{i} / \partial t$ (see limiting conditions (43)), so this term is also empty. Thus the zipper can be written as:

$$
Z\left(H_{i}^{*} H_{0 i}\right)=\left\{z_{1}, z_{2}\right\}=\left\{\begin{array}{l}
{\left[\left\{T_{i}, P_{i}, Q_{i}\right\},\left\{f^{i}, s^{i}, \mathbf{O}\right\}\right]} \\
{\left[\left\{F^{i} \backslash T_{i} \cap F^{j} \backslash T_{j}, S^{i} \backslash P_{i}, \mathbf{B}^{i}\right\},\left\{\tau_{i}, \varnothing, \mathbf{O}\right\}\right]}
\end{array}\right\},
$$

in which the qualities in $\mathrm{Z}$ are replaced by the interaction in consideration. Small-scale time element $\tau_{i}$ is assigned to the large-scale element $S^{i} \backslash P_{i}$, a space which cannot move (because the particle is isolated) and not shrink (because the radius of $S^{i}$ is by definition constant). So $z_{2}$ has no appearance and the appearance of the zipper can be written as:

$$
A\left(H_{i}^{*} H_{0 i}\right)=\sigma_{s^{i}} \text {. }
$$

Thus $H_{i}$ and $H_{0 i}$ generate an observable major particle $\sigma_{S^{i}}$, having geometry $s^{i}$ and charge $Q_{i}$; in the following we will write this shortly as $\sigma_{i}$. In this isolated situation, no space appears. In similar way particle $\sigma_{j}$ is generated.

As soon as neutral $\mathrm{H}$-unit $H_{0 i}$ starts to interact with the second neutral H-unit $H_{0 j}$, they will generate actual space. As we pointed out previously (Backerra, 2014), which will be repeated shortly in the next sections, these $\mathrm{H}$-units move accelerated towards each other and the particles will be taken in this movement. As soon as this happens, $\mathbf{E}_{i}$ changes accelerated, so in equation (44), in the first zip $\partial \mathbf{E}_{i} / \partial t$ changes linear and so in the second zip $\nabla \times \mathbf{B}^{i}$ changes also linear. So the vector fields in the zipper are not zero any more and zipper (45) is no longer valid. Consequently, the small-scale time element $\tau_{i}$ is not assigned to the large-scale element, but to the magnetic term (being independent of space). Then appearance (46) contains not only a particle, but also a space:

$$
A\left(H_{i}^{*} H_{0 i}\right)=\left\{\sigma_{i},\left(\Theta_{S^{i} \backslash P_{i}}^{\mathbf{B}^{i}}\right)\right\},
$$

being particle $\sigma_{i}$ and actual unobservable, magnetized, extended space $\Theta_{S^{i} \backslash P_{i}}^{\mathbf{B}^{i}}$, and similar for $H_{j}{ }^{*} H_{0 j}$, generating the appearances $\sigma_{j}$ and $\Theta_{S^{j} \backslash P_{j}}^{\mathbf{B}^{j}}$. Thus the interactions $H_{i}{ }^{*} H_{0 i}$ and $H_{j}{ }^{*} H_{0 j}$, each generating a particle, start to generate as well magnetized spaces as soon as the neutral $\mathrm{H}$-units start to overlap each other. This is a third interaction, being $H_{0 i}{ }^{*} H_{0 j}$, which will be considered in the next section.

\subsection{Gravity between the Two Particles}

The accelerated movement is described by a third zipper (after (45) and a similar zipper for indices $j$ and $0 j$, concerning the interaction between the two neutral H-units. At the start, the distance of their major points is such, that they have a relatively very small overlapping area, which is not overlapping the two magnetic spaces around the particles. The positions of $H_{0 i}$ and $H_{0 j}$ with respect to each other corresponds with space case 7 . Because we want to describe an accelerated movement, we take time case 2. Then the zipper for $H_{0 i}{ }^{*} H_{0 j}$ contains only one zip, which can be written as:

$$
Z\left(H_{0 i}{ }^{*} H_{0 j}\right)=\left\{z_{2}\right\}=\left\{\left\{F^{0 i} \backslash T_{0 i} \cap F^{0 j} \backslash T_{0 j}, S^{0 i} \cap S^{0 j}\right\},\left\{\tau_{0 i} \cap \tau_{0 j}, \varnothing\right\}\right\} .
$$


Small-scale time element $\tau_{0 i} \cap \tau_{0 j}$ is independent of space, so it will be assigned to the large-scale element and thus describes an accelerated change of the major space intersection $S^{0 i} \cap S^{0 j}$. This space is not observable because the corresponding small-scale space element is empty. Then the appearance (see section 2.5) can be written as:

$$
A\left(H_{0 i}{ }^{*} H_{0 j}\right)=\left(\Theta^{0}{ }_{S^{0 i} \cap S^{0 j}}\right) .
$$

This is an actual, unobservable, neutral, major free space, having the disc-like shape of two spherical segments. The indicated change $\tau_{0 i} \cap \tau_{0 j}$ can only be an accelerated change of the radii of $S^{0 i}$ and $S^{0 j}$, or a change of the distance between $P_{0 i}$ and $P_{0 j}$. A change of the radii is not possible because the size of an H-unit is constant, and so the H-units move from or towards each other. If moving from each other, the case would be finished in a short time, so we consider the second possibility: $P_{0 i}$ and $P_{0 j}$ move in an accelerated way towards each other. Consequently, the distance between the particles $\sigma_{i}$ and $\sigma_{j}$, existing in connection with $P_{0 i}$ and $P_{0 j}$, also decreases in an accelerated way and thus they move accelerated towards each other. This is identified with gravity.

Major free space $\Theta^{0}{ }_{S^{0 i} \cap S^{0 j}}$ exists in between of the particles, not covering them, nor overlapping a part of their magnetic spaces. As $P_{0 i}$ and $P_{0 j}$ move accelerated towards each other, the neutral space grows larger, so more and more potential energy of the neutral H-units is converted into actual energy.

This process might be circumscribed as gravity produces actual space.

\subsection{Involvement of Magnetism}

As soon as $\Theta^{0}{ }_{S^{0 i} \cap S^{0 j}}$ is large enough to overlap the magnetic spaces $\Theta_{S^{i} \backslash P_{i}}^{\mathbf{B}^{i}}$ and $\Theta_{S^{j} \backslash P_{j}}^{\mathbf{B}^{j}}$, each on one side, electromagnetism will be involved in the attracting process. From that moment, the interaction occurs between all four H-units. Because our zipper is suited only for the interaction of two H-units, we will consider each of the possible interactions between the four H-units separately; after that, we will consider them as a cluster of interactions, under the restriction that the energy of the complete phenomenon has to be equal to the sum of the isolated $\mathrm{H}$-events.

We will start with marked H-unit $H_{i}$ and neutral one $H_{0 j}$ (note that the index of the neutral H-unit now is $j$ ). The zipper for $H_{i}{ }^{*} H_{0 j}$ in time case 2 (for $T_{0 i}<T_{0 j}$ ) and space case 7 is:

$$
Z\left(H_{i}^{*} H_{0 j}\right)=\left\{z_{2}\right\}=\left\{\left\{F^{0 i} \cap F^{0 j} \backslash T_{0 j}, S^{i} \cap S^{0 j}, \mathbf{B}^{i}\right\},\left[\tau_{i} \cap \tau_{0 j}, \varnothing, \nabla \times \mathbf{B}^{i}\right]\right\} .
$$

Compared with Equation (48), a magnetic field and a magnetic derivations field are added. Small-scale mark element $\nabla \times \mathbf{B}^{i}$ is independent of space and, according to the limiting conditions (43), equal to $\partial \mathbf{E}_{i} / \partial t$. Because $\sigma_{i}$ moves accelerated, $\mathbf{E}_{i}$ changes with the second derivative of time, so $\partial \mathbf{E}_{i} / \partial t$ with the first derivative, which cannot be described by $\tau_{i} \cap \tau_{0 j}$. So the magnetic field derivative will be replaced by a zero vector field and the small-scale time element will be assigned to the large-scale element, describing an accelerated change of the major space. The appearance can be written as:

$$
A\left(H_{i}^{*} H_{0 j}\right)=\left(\Theta_{S^{i} \cap S^{0 j}}^{\mathbf{B}^{i}}\right) .
$$

The same is valid for the mirrored interaction $H_{j}{ }^{*} H_{0 i}$, generating the appearance:

$$
A\left(H_{j}^{*} H_{0 i}\right)=\left(\Theta_{S^{j} \cap S^{0 i}}^{\mathbf{B}^{j}}\right) .
$$

Thus the actual energy of the neutral space $\Theta^{0} S^{0 i} \cap S^{0 j}$ is enriched with the actual energy of the two magnetized spaces $\Theta_{S^{i} \cap S^{0 j}}^{\mathbf{B}^{i}}$ and $\Theta_{S^{j} \cap S^{0 i}}^{\mathbf{B}^{j}}$. This implies that the energy of the gravitational process increases as soon as the magnetized spaces start to play a role in the interaction. 


\subsection{Involvement of Electricity}

There will be a moment that the two particles have come so close to each other, that their magnetized major spaces $\Theta_{S^{i} \backslash P_{i}}^{\mathbf{B}^{i}}$ and $\Theta_{S^{j} \backslash P_{i}}^{\mathbf{B}^{j}}$ start to overlap each other. From that moment, interaction $H_{i}{ }^{*} H_{j}$ will be involved, the last of the six possible ${ }^{i}$ interactions between the four H-units. We will consider this interaction for time case 2 and space case 7 (so $S^{i} \cap S^{j} \neq \varnothing, \quad p_{i} \notin S^{j}$ and $p_{j} \notin S^{i}$ ). Then the zipper contains only zip $z_{2}$ :

$$
Z(t, \mathbf{x}, q)=\left\{z_{2}\right\}=\left[\left\{F^{i} \backslash T_{i} \cap F^{j} \backslash T_{j}, S^{i} \cap S^{j}, \mathbf{B}^{i}+\mathbf{B}^{j}\right\},\left\{\tau_{i} \cap \tau_{j}, \varnothing, \widetilde{\nabla} \times \widetilde{\mathbf{B}}^{i} \propto \widetilde{\nabla} \times \widetilde{\mathbf{B}}^{j}\right\}\right] .
$$

Using a field condition (see (43)), the small-scale mark element can be written as $\partial \widetilde{\mathbf{E}}_{i} / \partial \tilde{t} \propto \partial \widetilde{\mathbf{E}}_{j} / \partial \tilde{t}$ and this term transforms into an actual energetic $\mathrm{H}$-event only if it is accelerated changing. Because the particle moves accelerated, $\mathbf{E}_{i}$ changes with the second derivative of time, so $\partial \mathbf{E}_{i} / \partial t$ with the first derivative, which cannot be described by $\tau_{i} \cap \tau_{0 j}$. Thus the small-scale magnetic term will be replaced by an empty set. Then the small-scale time element $\tau_{i} \cap \tau_{0 j}$ will be assigned to the large-scale element, so describing an accelerated change of the major space intersection. This change may be a shrinking or growing of $S^{i} \cap S^{j}$. It is not observable, because the small-scale space element is empty. Then the appearance can be written as

$$
A(t, \mathbf{x}, q)=\left(\begin{array}{c}
\Theta_{S^{i} \cap S^{j}}^{\mathbf{B}^{i}+\mathbf{B}^{j}} \\
S^{\prime}
\end{array}\right.
$$

being and an actual, unobservable, magnetized, major extended space, changing in an accelerated way.

If $Q_{i}=Q_{j}$, the field lines of $\mathbf{E}_{i}$ and $\mathbf{E}_{j}$ inside space $\Theta_{S^{i} \cap S^{j}}^{\mathbf{B}^{i}+\mathbf{B}^{j}}$ have opposite components in the direction of the line segment $P_{i} P_{j}$, and so the actual energy density of the marked space will be smaller than in case only one electric field exists. However, the actual energy density of marked spaces is a constant, so to raise this decreasing density, $\Theta_{S^{i} \cap S^{j}}^{\mathbf{B}^{i}+\mathbf{B}^{j}}$ will shrink, implying that $P_{i}$ and $P_{j}$ move away from each other. If $Q_{i}=-Q_{j}$, the field lines have equal components in the direction of $P_{i} P_{j}$, and so the actual energy of the marked space will be larger than in case only one electric field exists. However, the actual energy density of marked spaces is a constant, so to reduce this increasing density, $\Theta_{S^{i} \cap S^{j}}^{\mathbf{B}^{i}+\mathbf{B}^{j}}$ will grow, implying that $P_{i}$ and $P_{j}$ move towards each other. Thus equal charges are repulsing, and opposite charges are attracting each other.

As soon as the major points $P_{i}$ and $P_{j}$ approach each other far enough to enter each others major spaces, space case 6 is valid; then the zipper contains one zip more, being $z_{3}$. Because $z_{2}$ is the same as in (53), we write only the new one:

$$
z_{3}=\left[\left\{T_{i}, P_{i}, Q_{i}\right\},\left\{\varnothing, \varnothing, \partial \widetilde{\mathbf{E}}_{i} / \partial \tilde{t} \propto \widetilde{\nabla} \times \widetilde{\mathbf{B}}^{j} \propto \widetilde{\nabla} \times \widetilde{\mathbf{E}}_{i} \propto \partial \widetilde{\mathbf{B}}^{j} / \partial \tilde{t}\right\}\right] .
$$

The small-scale field term is independent of time and space, so it will be assigned to the large-scale element, where it appears as an electromagnetic vector in major point $P_{i}$. This vector does not add energy because there is no space. The point is storing the actual energy of the charge, being unobservable because the small-scale geometry is empty. The appearance (54) is enriched with an unobservable charged point, so:

$$
A\left(H_{i}^{*} H_{j}\right)=\left\{\left(\Pi_{P_{i}, Q_{i}}\right),\left(\Theta_{S^{i} \cap S^{j}}^{\mathbf{B}^{i}+\mathbf{B}^{j}}\right)\right\} .
$$

Thus, as soon as $P_{i}$ and $P_{j}$ enter each others major spaces, a charge enters the energetic scene, together with the already existing magnetized space. 


\subsection{The Involvement of the Neutral Pellicles}

As the last detail of the interaction between the four H-units, we will consider interaction $H_{0 i} * H_{0 j}$ once more. The space cases, having started with space case 7 at the start, pass subsequently through cases 6,5 and 4 . For these three space cases, time case 2 is used, so the time zipper:

$$
Z(t)=\left\{\varnothing,\left\{F^{i} \backslash T_{i} \cap F^{j} \backslash T_{j}, \tau_{i} \cap \tau_{j}\right\},\left\{T_{i}, \varnothing\right\}, \varnothing\right\} .
$$

Time zippers $z_{1}$ and $z_{4}$ are empty and so the appearances as well. In space cases 6,5 and $4, z_{3}=\left\{P_{i}, \varnothing\right\}$, which can generate no energy in combination with time zip $\left\{T_{i}, \varnothing\right\}$. Thus the subsequent zippers contain only zip $z_{2}$. Written for increasing overlapping of $S^{0 i}$ and $S^{0 j}$, the zipper in space case 6 is:

$$
Z_{6}(t, \mathbf{x})=\left\{z_{2}\right\}=\left[\left\{F^{i} \backslash T_{i} \cap F^{j} \backslash T_{j}, S^{i} \backslash P_{i} \cap S^{j} \backslash P_{j}\right\},\left\{\tau_{i} \cap \tau_{j}, \varnothing\right\}\right],
$$

in which the index of $Z$ indicates the number of the space case. The appearance is $\left(\Theta^{0}{ }_{S^{0 i} \cap S^{0 j}}\right)$, being the same unobservable, accelerating space as in (49), so nothing new. The zipper in space case 5 is:

$$
Z_{5}(t, \mathbf{x})=\left\{z_{2}\right\}=\left[\left\{F^{i} \backslash T_{i} \cap F^{j} \backslash T_{j}, S^{i} \backslash P_{i} \cap S^{j} \backslash P_{j}\right\},\left\{\tau_{i} \cap \tau_{j}, p_{i j}\right\}\right],
$$

and in space case 4 :

$$
Z_{4}(t, \mathbf{x})=\left\{z_{2}\right\}=\left[\left\{F^{i} \backslash T_{i} \cap F^{j} \backslash T_{j}, S^{i} \backslash P_{i} \cap S^{j} \backslash P_{j}\right\},\left\{\tau_{i} \cap \tau_{j}, p_{i} \cap p_{j}\right\}\right] .
$$

Now a change is described: in both cases minor particle $\pi_{p_{i} \cap p_{j}}$ appears. The minor particle pops up in equation (59) and starts to travel through the pellicle intersection in equation (60). As a consequence, no extended space appears any more in interaction $H_{0 i}{ }^{*} H_{0 j}$, because space $\left(\Theta^{0}{ }_{S^{0 i}} \cap S^{0 j}\right)$ reduces to the track of the minor particle. Thus, from the moment that space case 5 is reached, the gravitational process has ended. Particle $\pi_{p_{i} \cap p_{j .}}$ is neutral and has a small mass; its existence ends as soon as space case 4 is reached. We identify this H-event with a Higgs particle, suddenly appearing in the last phase of the gravitational process and existing only during the small interval of time $\tau_{i} \cap \tau_{j}$.

Summarizing, the introduction of electromagnetic features adds a second type of accelerated movement to the gravitational process, away or towards each other, depending on the signs of the charges with respect to each other. Because magnetized major spaces are smaller then neutral ones, this addition can appear only after the gravitational process has started. In its first phase, magnetic energy is added to the total process; in the second phase, also charge energy is added. The Higgs particle is generated immediately after the gravitational process has ended and exists only during an extreme small period of time.

The results as presented in this section, can explain the instability in the experimentally observed constants of gravity. If we take the radius of $S^{0 i}$ as a first estimation as $R_{0 i}=100 \times R_{i}$, then the volume of $S^{0 i}$ is $10^{6}$ larger than that of $S^{i}$, and, taking the energy density of a marked space as 100 times that of a neutral space, then the total additional actual energy of the marked space (counting with completely overlapping spaces) is about $10^{-4}$ times the total actual energy of the neutral space. The official value of $G$ is $6.673889 \times 10^{-11} \mathrm{~N} \cdot(\mathrm{m} / \mathrm{kg})^{2}$, but the 13 measured values analyzed in a study of Lisa Zyga (2015) range from approximately $6.672 \times 10^{-11} \mathrm{~N} \cdot(\mathrm{m} / \mathrm{kg})^{2}$ to $6.675 \times 10^{-11} \mathrm{~N} \cdot(\mathrm{m} / \mathrm{kg})^{2}$, which is a variation of about $10^{-4}$.

\subsection{Expansion of the Universe}

If the four H-units, as considered in the previous sections, would exist in an isolated system, their interactions would be impossible, because an increase of the total actual energy of the system is not allowed by the law of conservation of actual energy. If the system contains much more H-units, the increase of actual energy by the gravitational process can be compensated by another process. The possibility of repulsing of the same type of interaction is suited for this. To consider the possibility of an accelerated moving of $P_{0 i}$ and $P_{0 j}$ away from each other, we repeat (48):

$$
z_{2}(t, \mathbf{x})=\left\{\left\{F^{0 i} \cap F^{0 j} \backslash T_{0 j}, S^{0 i} \cap S^{0 j}\right\},\left\{\tau_{0 i} \cap \tau_{0 j}, \varnothing\right\}\right\} .
$$


The appearance $\left(\Theta_{S^{0 i} \cap S^{0 j}}^{0}\right)$, shortly written as $\Theta^{0}$, is an actual, unobservable neutral space, moving in an accelerated way such that its size and so its actual energy decreases. Adding two marked H-units to these two neutral H-units in a similar way as we did to describe gravity, we have a second cluster of four H-units, describing two charged particles $\sigma_{i}$ and $\sigma_{j}$. In this repulsing interaction, the marked spaces of these particles

are supposed to exist outside the reach of $\Theta^{0}$, but on the other hand, space $S^{0 i} \cap S^{0 j}$ is supposed to be large enough to contain $P_{0 i}$ and $P_{0 j}$. Then the particles move accelerated away from each other. Space $\Theta^{0}$ shrinks until it is empty and all actual space energy is converted back into potential energy. This could be expressed by saying that actual space is swallowed up by the expansion.

During the interaction, each of the H-units has an accelerated growing velocity with respect to the other one, as if they are launched with respect to each other. After the interaction has finished by losing contact between their major spaces, the velocity is not accelerating any more, but constant. So the H-units will continue to move away from each other, with the same velocity as they have reached. The particles $\sigma_{i}$ and $\sigma_{j}$ inside, move away from each other without ever being able to interact, and so their electromagnetic features stay out of the appearance. Thus the first phase of expansion is an accelerated period and, after the neutral spaces lost contact, switches over to a moving away from each other with a constant velocity.

We identify this H-event with the expanding universe, which in classical physics is considered as a separate phenomenon. According to twin physics, gravity and the expanding universe could be considered as two distinct aspects of one type of interaction.

\subsection{The Cluster of Interactions Considered Together}

Summarizing section 3, gravity is generated by space energy, whilst electric attraction and repulsion are generated by mark energy. The difference of gravity and electricity is in their range, resulting from the different sizes of neutral and marked major spaces. Because the range of electric attraction or repulsion is smaller than the range of gravity, spatial effects are allowed to act separately from marking effects.

Although attraction and expansion have the same origin, namely an accelerated change of a space, they show a striking difference. Gravity will be combined with electric repulsion or attraction as soon as the magnetic spaces around the particles are reached. Expansion, on the contrary, never combines with electromagnetic effects. At an astronomic scale, these two phenomena are adding to conserve the total actual energy of the universe by balancing their production and swallowing of space. If this happens with a conglomeration of $\mathrm{H}$-units, then clusters of gravitating H-units may develop, drifting apart from each other due to expansion.

\section{Overview of the Results of Twin Physics}

Until now, we obtained for each investigated case one or two zips describing H-events, so having actual energy; the remaining elements of the zipper were empty. If two zips appeared, we considered them in combination with each other, to get a view on complementarity. However, in this section we want to prepare our results for a comparison with those of the standard model, and therefore a series of zips is considered separately, keeping in mind that the story might be not complete without considering the complete zipper. By far not all possible cases are considered. With these rather brief examples, not going into the details, we try to evoke a feeling for the basic character of each zip. After that, in section 4.5, we will use these results to describe the decay of a neutron.

Interactions of two marked $\mathrm{H}$-units (called marked interactions) are indicated by $H_{i}{ }^{*} H_{j}$; interactions involving one neutral H-unit (mixed interactions) are indicated by $H_{i}{ }^{*} H_{0 i}$, and if both H-units are neutral (neutral interactions) by $H_{0 i} * H_{0 j}$. Bear in mind that neutral major as well as minor spaces (indicated by a zero index) are larger than marked spaces. In general the time and space cases are chosen such, that the zips show as much as possible, or that they are useful for describing the neutron decay. All time attributes are transformed to a physical space, so the tildes and square brackets are removed; for shortness, in general time elements will only be explained if indispensable. Most of the space attributes are transformed to a physical space; elements containing pellicle intersections are still written with square brackets, because usually this is a non-singular object. Possibly only in the last step of transformation the appearance of these objects is clear. The operations joining, linking and transforming in the mark zippers (41) and (42) are carried out as far as possible; as long as the transformation cannot be fully decided, the operators will remain in the zipper. 


\subsection{The First Zip}

Zip $z_{1}$ describes particles, but only in time case 1 and if the major spaces overlap each other such that the major points of space are inside the overlapping region. Then the appearance may be a spherical or slightly flattened major particle, having mass and eventually charge. It may be identified with black matter, a neutron, a proton, or an antiproton. This zip is essential in the decay process of the neutron (see section 4.5). Moreover this zip is the basis of the so-called 'strong force'.

4.1.1 For two neutral H-units in time case $1\left(T_{0 i} \cap T_{0 j}=T_{0 i}\right)$ and space case $1\left(P_{0 i} \cap P_{0 j}=P_{0 i}\right)$ :

$$
z_{1}\left(H_{0 i} * H_{0 j}\right)=\left\{\left\{T_{0 i}, P_{0 i}\right\},\left\{f^{0 i}, s^{0 i}\right\}\right\} .
$$

Appearance $A\left(z_{1}\right)$ is $\sigma_{s^{0 i}}$, being a major particle without electromagnetic features and having geometry $s^{0 i}$. Its radius is much larger than that of a neutron. It is identified with black matter.

4.1.2 For two marked H-units in time case $1\left(T_{i} \cap T_{j}=T_{i}\right)$ and space case $1\left(P_{i} \cap P_{j}=P_{i}\right)$ :

$$
z_{1}\left(H_{i}^{*} H_{j}\right)=\left\{\left\{T_{i}, P_{i},\left[\widetilde{Q}_{i} \propto \widetilde{Q}_{j}\right]\right\},\left\{f^{i}, s^{i},\left[\partial \widetilde{\mathbf{E}}_{i} / \partial \tilde{t} \propto \partial \widetilde{\mathbf{E}}_{j} / \partial \tilde{t}\right]\right\}\right\} .
$$

If in the considered system no other H-units exists to move to or from, then $\partial \widetilde{\mathbf{E}}_{i} / \partial \tilde{t}=\partial \widetilde{\mathbf{E}}_{j} / \partial \tilde{t}=\mathbf{O}$. Appearance $A\left(z_{1}\right)$ is $\sigma_{s^{i}}$, being a spherical major particle. If $Q_{i}=Q_{j}$, the particle is neutral and identified with a neutron. If $Q_{i}=Q_{j}$, the particle is charged and identified with a proton or an anti-proton. Mind that $\left[\widetilde{Q}_{i} \propto \widetilde{Q}_{i}\right]=\left[\widetilde{Q}_{i}\right]=Q_{i}$. 4.1.3 For one marked and one neutral H-unit in time case $1\left(T_{i} \cap T_{0 j}=T_{i}\right)$ and space case 8

$$
\left(P_{i} \cap P_{0 j}=P_{i}\right)
$$

$$
z_{1}\left(H_{i}^{*} H_{0 i}\right)=\left\{\left\{T_{i}, P_{i}, Q_{i}\right\},\left\{f^{i}, s^{i}, \partial \mathbf{E}_{i} / \partial t\right\}\right\} .
$$

If in the considered system no other H-units exists to move to or from, then $\partial \mathbf{E}_{i} / \partial t=\mathbf{O}$. Appearance $A\left(z_{1}\right)$ is $\sigma_{s i}$, being a major particle having geometry $s^{i}$. For positive $Q_{i}$, it is again identified with a proton and for negative charge with an antiproton. This zip is the basis of the strong force, because the neutral minor space $s^{0 i}$ supplies the connecting background of an atom, without generating attracting or repulsing electric field derivatives.

4.1.4 For two marked H-units in time case $1\left(T_{i} \cap T_{j}=T_{i}\right)$ and space case $2\left(P_{i} \in s^{i} \cap s^{j}\right.$ and $\left.P_{i} \cap P_{j}=\varnothing\right)$ :

$$
z_{1}\left(H_{i}^{*} H_{j}\right)=\left\{\left\{T_{i}, \varnothing,\left[\widetilde{Q}_{i} \propto \widetilde{Q}_{j}\right]\right\},\left\{f^{i}, s^{i} \cap s^{j},\left[\partial \widetilde{\mathbf{E}}_{i} / \partial \tilde{t} \propto \partial \widetilde{\mathbf{E}}_{j} / \partial \tilde{t}\right]\right\}\right\} .
$$

If in the considered system no other H-units exists to move to or from, then $\partial \widetilde{\mathbf{E}}_{i} / \partial \tilde{t} \propto \partial \widetilde{\mathbf{E}}_{j} / \partial \tilde{t}=\mathbf{O}$.

The difference with (63) is, that here the large-scale space element is empty. The appearance is the same, so a

neutron, a proton or an anti-proton, without a large-scale major point of space.

4.1.5 For one marked and one neutral H-unit in time case 2 (so $T_{i} \in f^{0 i}$ and $\tau_{i} \cap \tau_{0 i} \neq \varnothing$ ) and space case 9

$\left(P_{0 i} \in s^{i}\right.$ and $\left.P_{i} \cap P_{j}=\varnothing\right)$ :

$$
z_{1}\left(H_{i}^{*} H_{0 i}\right)=\left[\left\{\varnothing, \varnothing, Q_{i}\right\},\left\{\varnothing, s^{i}, \partial \mathbf{E}_{i} / \partial t\right\}\right] .
$$

This is a demonstration that the first zip in another time case than time case 1 has no appearance. The interaction generates no actual energy at all in this zip and there is no H-event.

\subsection{The Second Zip}

Zip $z_{2}$ generates actual energy for all interactions, if only the major spaces have a non-empty intersection and so can interact in general. The described H-events all are related to 'a large space', or to 'space to travel through' and in general they are changing with time. If the small-scale geometry is empty, the large-scale one appears as an actual, unobservable major extended space, which might be magnetized; it is active by changing its size or by turning around. If the small-scale geometry is not empty, the zip describes a particle, existing in the intersection of pellicles, having no charge and being considerably smaller than a major particle. In these cases, the major 
space is restricted to the track of the particle. Two types of gluons or a neutrino may appear. This zip is the basis for gravity, expansion, electric attraction and repulsion.

4.2.1 For two neutral H-units in time case 1 (so $\left.T_{0 i} \cap T_{0 j}=T_{0 i}\right)$ and space case $1\left(P_{0 i} \cap P_{0 j}=P_{0 i}\right)$ :

$$
z_{2}\left(H_{0 i} * H_{0 j}\right)=\left[\left\{F^{0 i} \backslash T_{0 i}, S^{0 i} \backslash P_{0 i}\right\},\left\{\tau_{0 i}, p_{0 i}\right\}\right] .
$$

Appearance $A\left(z_{2}\right)$ is $\pi_{p_{0 i}}$, being a minor particle, having a small mass (compared with a major particle), no charge, and traveling with a constant velocity over the surface of $s^{0 i}$ (see section 4.1.1). It is identified with a gluon.

4.2.2 For two marked H-units in time case 1 (so $T_{i} \cap T_{j}=T_{i}$ ) and space case 1 (so $P_{i} \cap P_{j}=P_{i}$ ):

$$
z_{2}\left(H_{i}^{*} H_{j}\right)=\left[\left\{F^{i} \backslash T_{i}, S^{i} \backslash P_{i}, \mathbf{B}^{i}+\mathbf{B}^{j}\right\},\left\{\tau_{i}, p_{i},\left[\widetilde{\nabla} \times \widetilde{\mathbf{B}}^{i} \propto \widetilde{\nabla} \times \widetilde{\mathbf{B}}^{j}\right]\right\}\right] .
$$

Mind that $\mathbf{B}^{i}+\mathbf{B}^{j}$ cannot be reduced to zero, because each major magnetic field is by definition unique (see section 2.3). Appearance $A\left(z_{2}\right)$ is $\pi_{p_{i}}$, being a minor particle, having no charge, traveling with a constant velocity through $p_{i}$ over the surface of $s^{i}$ (see section 4.1.2). Like in the previous example, this is identified with a gluon.

4.2.3 For one marked and one neutral $\mathrm{H}$-unit in time case 1 (so $T_{i} \cap T_{0 i}=T_{i}$ ) and space case 8 (so $P_{i} \cap P_{0 j}=P_{i}$ ):

$$
z_{2}\left(H_{i}^{*} H_{0 i}\right)=\left[\left\{F^{i} \backslash T_{i}, S^{i} \backslash P_{i}, \mathbf{B}^{i}\right\},\left\{\tau_{i}, \varnothing, \nabla \times \mathbf{B}^{i}\right\}\right] .
$$

If in the considered system no other H-units exists, a movement is not possible and so $\nabla \times \mathbf{B}^{i}=\mathbf{O}$ (because of conditions (43)), so the small-scale time element $\tau_{i}$ will be assigned to the large-scale element of $z_{2}$. But because the major space cannot move, nor grow or shrink, there is no appearance. If at least one H-unit more exists in the considered system, a movement is possible, the magnetic field term is not a zero field and $\tau_{i}$

indicates a change in the small-scale element. Then the appearance $A\left(z_{2}\right)$ is $\left(\Theta_{S^{i} \backslash P_{i}}^{\left(\mathbf{B}^{i}\right)}\right)$, in which the brackets

around $\mathbf{B}^{i}$ indicate a changing field. This can be identified with an actual, unobservable, major extended space, carrying a changing magnetic field.

4.2.4 For one marked and one neutral H-unit in time case 2 (so $T_{i} \in f^{0 i}$ and $\tau_{i} \cap \tau_{0 i} \neq \varnothing$ ) and space case 9

$\left(P_{0 i} \in s^{i}\right.$ and $\left.P_{i} \cap P_{0 i}=\varnothing\right)$, and also for space case $11\left(s^{i} \subset s^{0 i}\right.$ and $\left.P_{0 i} \notin s^{i}\right)$ :

$$
z_{2}\left(H_{i}^{*} H_{0 i}\right)=\left[\left\{F^{i} \backslash T_{i} \cap F^{0 i} \backslash T_{0 i}, S^{i} \backslash P_{i}, \mathbf{B}^{i}\right\},\left\{\tau_{i} \cap \tau_{0 i}, \varnothing, \nabla \times \mathbf{B}^{i}\right\}\right] .
$$

Small-scale time element $\tau_{i} \cap \tau_{0 i}$ indicates an accelerated change; this is possible if $S^{i} \backslash P_{i}$ turns around $P_{0 i}$.

Then appearance $A\left(z_{2}\right)$ is $\left(\Theta_{S^{i} \backslash P_{i}}^{\left(\mathbf{B}^{i}\right)}\right)$, being an actual, unobservable, major extended space, carrying a

quadratic changing magnetic field. But because of field conditions (43), this would be possible only if also $\partial \mathbf{E}_{i} / \partial t$ would change quadratic and so if $\mathbf{E}_{i}$ would change in the third order. We cannot identify this with experimental results, so we suppose that the small-scale field is not appearing.

4.2.5 For two marked H-units in time case 2 (so $T_{i} \in f^{j}$ and $\tau_{i} \cap \tau_{j} \neq \varnothing$ ) and space case 2 (so $P_{i} \in s^{i} \cap s^{j}$ and $\left.P_{i} \cap P_{j}=\varnothing\right)$ :

$$
z_{2}\left(H_{i}^{*} H_{j}\right)=\left[\left\{F^{i} \backslash T_{i} \cap F^{j} \backslash T_{j}, S^{i} \backslash P_{i} \cap S^{j} \backslash P_{j}, \mathbf{B}^{i}+\mathbf{B}^{j}\right\},\left\{\tau_{i} \cap \tau_{j}, p_{i} \cap p_{j}, \nabla \times \mathbf{B}^{i} \propto \nabla \times \mathbf{B}^{j}\right\}\right],
$$

with the large-scale element written above the small-scale one. Appearance $A\left(z_{2}\right)$ is $\pi_{p_{i} \cap p_{j}}$, being a minor particle, having a small mass and no charge, and traveling accelerated through $p_{i} \cap p_{j}$ along the border of $s^{i} \cap s^{j}$. The H-event could again be identified with a gluon, but there is a difference with the previous two: This one accelerates, as if being launched. If it continues its existence, it has a constant velocity close to the velocity of light (see Backerra 2012). This will be identified with a neutrino. 
4.2.6 For two neutral H-units in time case 2 (so $T_{0 i} \in f^{0 j}$ and $\tau_{0 i} \cap \tau_{0 j} \neq \varnothing$ ) and space case 7 (so $S^{i} \cap S^{j} \neq \varnothing, \quad p_{i} \notin S^{j}$ and $\left.p_{j} \notin S^{i}\right)$ :

$$
z_{2}\left(H_{0 i} * H_{0 j}\right)=\left\{\left\{F^{0 i} \backslash T_{0 i} \cap F^{0 j} \backslash T_{0 j}, S^{0 i} \cap S^{0 j}\right\},\left\{\tau_{0 i} \cap \tau_{0 j}, \varnothing\right\}\right\} .
$$

The small-scale time element $\tau_{0 i} \cap \tau_{0 j}$ is assigned to the large-scale one. The intersection of major spaces has the possibility to change, by growing or shrinking. Appearance $A\left(z_{2}\right)$ is $\left(\Theta_{S^{0 i} \cap S^{0 j}}^{0 j}\right)$, being an actual, unobservable, major extended space, changing accelerated. Thus the major points of space $P_{0 i}$ and $P_{0 j}$ move accelerated towards or from each other. The first possibility is identified with gravity, the second with expansion Note that both phenomena are observable only if major particles exist in the centre of the neutral $\mathrm{H}$-units, so if two marked $\mathrm{H}$-units are involved (see section 3).

4.2.7 For two marked H-units in time case 2 (so $T_{i} \in f^{j}$ and $\tau_{i} \cap \tau_{j} \neq \varnothing$ ) and space case 7 (so $S^{i} \cap S^{j} \neq \varnothing$, $p_{i} \notin S^{j}$ and $\left.p_{j} \notin S^{i}\right)$ :

$$
z_{2}\left(H_{i}^{*} H_{j}\right)=\left\{\left\{F^{i} \backslash T_{i} \cap F^{j}, S^{i} \cap S^{j}, \mathbf{B}^{i}+\mathbf{B}^{j}\right\},\left\{\tau_{i} \cap \tau_{j}, \varnothing,\left[\widetilde{\nabla} \times \widetilde{\mathbf{B}}^{i} \propto \widetilde{\nabla} \times \widetilde{\mathbf{B}}^{j}\right]\right\}\right\} .
$$

Appearance $A\left(z_{2}\right)$ is $\left(\Theta_{S^{i} \cap S^{j}}^{\mathbf{B}^{i}+\mathbf{B}^{j}}\right)$, being an actual, unobservable, magnetized major extended space, changing in an accelerated way. Because of field conditions (43), the small-scale mark element appears only if $\partial \widetilde{\mathbf{E}}_{i} / \partial \tilde{t} \propto \partial \widetilde{\mathbf{E}}_{j} / \partial \tilde{t}$ would change quadratic, and thus $\mathbf{E}_{i} \propto \mathbf{E}_{j}$ in the third order, so we

suppose that this field term does not appear. As pointed out in section (3.4), if $Q_{i}=Q_{j}$, the H-units repel each other; if $Q_{i}=Q_{j}$, they attract each other. This is identified with electric repulsion and attraction, respectively. Like in the previous example, this is only observable if two more (neutral) $\mathrm{H}$-units are involved.

4.2.8 For one marked and one neutral H-unit in time case 4 (so $T_{i} \in \tau_{0 i}$ for $T_{0 i}>T_{0}$, and space case 12 (so $P_{i} \in p_{0 i}$ ):

$$
z_{2}\left(H_{i}^{*} H_{0 i}\right)=\left[\left\{F^{i} \backslash T_{i} \cap F^{0 i} \backslash T_{0 i}, S^{i} \backslash P_{i} \cap S^{0 i} \backslash P_{0 i}, \mathbf{B}^{i}\right\},\left\{\varnothing, p_{i} \cap p_{0 i}, \nabla \times \mathbf{B}^{i}\right\}\right] .
$$

Appearance $A\left(z_{2}\right)$ is $\pi_{p_{i} \cap p_{0 i}}$, being a minor particle, traveling with a constant velocity through $p_{i} \cap p_{0}$. The field term $\nabla \times \mathbf{B}^{i}$ is independent of space. Depending on the remaining part of the zipper, it might be a gluon or a neutrino.

4.2.9 For one marked and one neutral H-unit in time case 1 (so $T_{i} \cap T_{0 i}=T_{i}$ ) and space case 13 (so $s^{i} \subset S^{0 i}$ and $\left.P_{0 i} \notin S^{i}\right)$, is:

$$
Z\left(H_{i}^{*} H_{0 i}\right)=\left\{z_{2}\right\}=\left\{\left\{F^{i} \backslash T_{i}, S^{i} \backslash P_{i}, \mathbf{B}^{i}\right\},\left\{\tau_{i}, \varnothing, \nabla \times \mathbf{B}^{i}\right\}\right\} .
$$

The appearance is non-empty if $\nabla \times \mathbf{B}^{i}$ changes linear, so if $\partial \mathbf{E}_{i} / \partial t$ changes linear, then $\mathbf{E}_{i}$ changes with the second order and thus the generated H-event moves accelerated. The H-units have eccentric positions with respect of each other, so $P_{i}$ and $P_{0 i}$ may move accelerated towards or from each other, or turn around each

other. Appearance $A\left(z_{2}\right)$ is $\left(\Theta_{S^{i} \backslash P_{i}}^{\mathbf{B}^{i}}\right)$, being an unobservable, magnetized major extended space. We will meet this case again in section 4.5 , considering the neutron decay.

\subsection{The Third Zip}

Zip $z_{3}$ is in general empty if the major points of the two $\mathrm{H}$-units are coinciding. Here we will consider only mixed interactions, generating an electron.

4.3.1 For one marked and one neutral H-unit in time case 5 (so $T_{i} \in F^{j}$ and $F^{i} \cap \tau_{j}=\varnothing$ ) and space case 10 (so $\left.P_{0 i} \in p_{i}\right)$ : 


$$
z_{3}\left(H_{i}^{*} H_{0 i}\right)=\left[\left\{T_{i}, P_{i}, Q_{i}\right\},\left\{\varnothing, \varnothing, \partial \mathbf{E}_{i} / \partial t+\nabla \times \mathbf{E}_{i}\right\}\right] .
$$

In this case, the small-scale time and space elements are empty, so small-scale mark element $\partial \mathbf{E}_{i} / \partial t+\nabla \times \mathbf{E}_{i}$ is independent of time and space. Appearance $A\left(z_{3}\right)$ is $\left(\Pi_{Q_{i}}\right)$, being an unobservable, charged point particle, storing the actual energy of $Q_{i}$ and carrying a vector of electromagnetic field $\partial \mathbf{E}_{i} / \partial t+\nabla \times \mathbf{E}_{i}$. Previously we identified this particle with an anti-photon (see Backerra, 2015), because in that context it acted as a counterbalance for the photon.

For $T_{i}<T_{0 i}$, the zip is empty.

4.3.2 For one marked and one neutral H-unit in time case 4 (so $T_{i} \in \tau_{0 j}$ for $T_{i}>T_{0 i}$ ) and space case 12 $\left(P_{i} \in p_{0 i}\right)$ :

$$
z_{3}\left(H_{i}^{*} H_{0 i}\right)=\left[\left\{T_{i}, P_{i}, Q_{i}\right\},\left\{f^{i} \cap \tau_{0 i}, s^{i} \cap p_{0 i}, \partial \mathbf{E}_{i} / \partial t+\nabla \times \mathbf{E}_{i}\right\}\right] .
$$

Major point $P_{i}$ exists inside the small-scale geometry $s^{i} \cap p_{0 i}$, turning together with a constant velocity around $P_{0 i}$ over the neutral pellicle $p_{0 j}$. Appearance $A\left(z_{3}\right)$ is $\delta_{s^{i}} \cap p_{0 i}$, being a dot particle inside the pellicle, having a small mass, a charge $Q_{i}$, and by definition shaped as a small sphere (see section 2.5); inside this particle, an electromagnetic field exists. It is identified with an electron.

For $T_{i}<T_{0 i}$, the zip is empty.

\subsection{The Fourth Zip}

Zip $z_{4}$ is empty in cases with coinciding major points. Here we will consider only a photon.

Then we need the time zipper in case 5 (so $T_{i} \in F^{j}$ and $\tau_{j} \cap F^{i}=\varnothing$, for $T_{i}<T_{0 i}$ ), which is $\left\{\varnothing,\left\{F^{i} \backslash T_{i} \cap F^{j}, \varnothing\right\}, \varnothing,\left\{T_{0 i}, \varnothing\right\}\right\}$. The space case is similar, so $P_{i} \cap S^{0 i} \neq \varnothing$ and $P_{0 i} \cap S^{i} \neq \varnothing$, but $p_{i} \cap p_{0 i}=\varnothing$. For one marked and one neutral H-unit in time case 5 and space case 13:

$$
z_{4}\left(H_{i} * H_{0 i}\right)=\left[\left\{T_{0 i}, P_{0 i}, \mathbf{b}^{i}\right\},\left\{\varnothing, \varnothing, \nabla \times \mathbf{B}^{i}+\partial \mathbf{B}^{i} / \partial t\right\}\right] .
$$

Mind that in our previous paper (Backerra 2015, section 7, equation (63)) the photon is described by the third zip in equation (64) instead of the fourth, because the major points of time were taken oppositely upon the time axis (so $T_{i}>T_{0 i}$ ). The small-scale mark element is independent of time and space. Appearance $A\left(z_{4}\right)$ is $\left(\Pi_{\mathbf{b}^{i}}\right)$, being an unobservable, neutral, massless point particle, having a magnetic vector $\mathbf{b}^{i}$ attached, and an electromagnetic vector. The generated particle is identified with a photon, the elementary particle without mass and charge, having an unobservable major location and carrying observable electromagnetic energy through space, with a constant velocity (see Backerra, 2015, section 7.1). The photon travels with a constant velocity around $P_{i}$, so it acts as a clock.

For $T_{i}>T_{0 i}$, the zip is empty.

\subsection{Decay of the Neutron}

A free neutron is known to decay into a proton, an electron and a neutrino. We will try to obtain a proper description of this process by considering three H-units, one being neutral and two oppositely charged, in subsequently four steps. Each of the three possible interactions between two $\mathrm{H}$-units will be considered separately and we will connect them by using the law of conservation of actual energy. In principle all combinations of time cases with space cases are allowed, if only they are compatible with each other and if the generated actual energy matches with the conservation law.

The neutral H-unit is $H_{0}$; the marked ones are $H_{i}$ and $H_{j}$, having charges $Q_{i}$ and $Q_{j}$, respectively, with $Q_{i}=-Q_{j}$ and $Q_{i}$ positive. The three possible interactions are written as $H_{i}{ }^{*} H_{j}, H_{i}^{*} H_{0}$ and $H_{j} * H_{0}$. Their sets of appearances are indicated by $A_{i j}, A_{i 0}$ and $A_{j 0}$, respectively.

Step 1: At the start we have a closed mathematical system containing these three $\mathrm{H}$-units.

The two marked ones, $H_{i}$ and $H_{j}$, are considered as coinciding in time case 1 and space case 1 (so $T_{i} \cap T_{j}=T_{i}$ and $P_{i} \cap P_{j}=P_{i}$ ). Together they exist inside $S^{0}$, which is the major space of $H_{0}$, close to the border. Then the two non-zero zips of our first considered interaction $H_{i}{ }^{*} H_{j}$ appear together as one H-event (see (63) and (68)): 


$$
A_{i j}\left(z_{1}, z_{2}\right)=\left\{\sigma_{s^{i}}, \pi_{p_{i}}\right\} .
$$

This is a neutron, written as $\sigma_{i j}^{0}$ (indices $i j$ indicate the involved H-units and 0 the charge), with a gluon $\pi_{i j}$ traveling over its surface with a constant velocity.

The appearance of our second considered interaction, $H_{i}{ }^{*} H_{0}$, in time case 1 (so $T_{i} \cap T_{0 i}=T_{i}$ ) and space case 13 (so $s^{i} \subset S^{0 i}$ and $P_{0 i} \notin S^{i}$ ) is (see (75)):

$$
A_{i 0}\left(H_{i}^{*} H_{0}\right)=\left(\Theta_{S^{i} \backslash P_{i}}^{\mathbf{B}^{i}}\right) .
$$

This is an unobservable, magnetized major extended space, written as $\Theta_{i 0}^{\mathbf{B}^{i}}$, moving with a constant velocity towards or from $P_{0}$. If the $\mathrm{H}$-units move away from each other, the case ends if the contact is lost and nothing will happen. So we consider them as moving towards each other.

Our third considered interaction, $H_{j}{ }^{*} H_{0}$, could in principle appear likewise, but the last step of transforming the zipper is not allowed by the law of conservation of actual energy, because then the actual energy of $\Theta_{i 0}^{\mathbf{B}^{\mathbf{q}}}$ would be doubled, and by definition the energy density of space is a constant.

Then the actual energy of the total system can be written as:

$$
E_{1}\left(H_{i}^{*} H_{j}^{*} H_{0}\right)=E\left(H_{i}^{*} H_{j}\right)+E\left(H_{i}^{*} H_{0}\right)
$$

in which the index 1 of $E$ indicates "before the interaction", and so with (79) and (80) as:

$$
E_{1}\left(H_{i}^{*} H_{j} * H_{0}\right)=E\left(\left(\sigma_{i j}^{0}+\pi_{i j}\right)+\Theta_{i 0}^{\mathbf{B}^{i}}\right) .
$$

Step 2: As the neutron moves linear towards $P_{0}$, there will be a moment that $P_{j}$ (still coinciding with $P_{i}$ ) reaches neutral pellicle $p_{0}$. Then $H_{j} * H_{0}$ can generate actual energy in space case 12 (so $P_{j} \in p_{0}$ ). We choose the time case similar to the space case: $T_{j} \in \tau_{0} \quad\left(\right.$ for $T_{j}>T_{0}$ ), so time case 4 .

The zipper is (see (74) and (77)):

$$
Z\left(H_{j}^{*} H_{0}\right)=\left\{z_{2}, z_{3}\right\}=\left\{\begin{array}{l}
{\left[\left\{F^{j} \backslash T_{j} \cap F^{0}, S^{j} \backslash P_{j} \cap S^{0} \backslash P_{0}, \mathbf{B}^{j}\right\},\left\{\varnothing, p_{j} \cap p_{0}, \nabla \times \mathbf{B}^{j}\right\}\right]} \\
{\left[\left\{T_{j}, P_{j}, Q_{j}\right\},\left\{f^{j} \cap \tau_{0}, s^{j} \cap p_{0}, \nabla \times \mathbf{E}_{j}\right\}\right]}
\end{array}\right\}
$$

and the appearance is:

$$
A_{j 0}\left(z_{2}, z_{3}\right)=\left\{\pi_{p_{j} \cap p_{0}}, \delta_{s^{j} \cap p_{0}}\right\} .
$$

The first element is a minor particle, traveling with a constant velocity through $p_{j} \cap p_{0}$, being a small circular track inside the relatively large neutral pellicle $p_{0}$. This track is the border of minor space $s^{j} \cap p_{0}$, containing $P_{j}$ in its centre; the transformation of this object is a dot particle, being identified with an electron $e_{j 0}^{-}$(see section 4.3.2). Its small-scale time element indicates that it moves with a constant velocity and this can only be a movement around $P_{0}$. The electron exists in the middle of the circulating minor particle, so the second particle is identified with a neutrino $v_{j 0}$, having a small mass, no charge and being distinct from a gluon (because it does not glues to the surface of a major particle). Then the actual energy of (only this) interaction can be written as:

$$
E\left(H_{j}^{*} H_{0}\right)=E\left(e_{j 0}^{-}+v_{j 0}\right)
$$

Step 3: Because $H_{j}$ is involved in generating the electron and the neutrino, interaction $H_{i}{ }^{*} H_{j}$ has less potential energy available to generate the neutron, but this requires all its potential energy. The only possibility to reduce the generated actual energy is a shift of $P_{i}$ and $P_{j}$, leading to space case 2. Supposing that the time shifts in the same way, we reach time case 2 . In time case 2 , no particle can be generated, so the actual energy of $\sigma_{i j}^{0}$ transforms back to potential energy and the neutron annihilates; it is not clear yet if the gluon annihilates also, or continues its existence in another way. The relatively large decrease of the actual energy in $z_{1}$ is not immediately violating the energy law, because only after the flying time $f^{i}$ (which is an extended present) this change has to be described in zip $z_{1}$, giving the opportunity to rearrange the remaining interactions. 
As a consequence of the loss of the neutron energy, interaction $H_{i}^{*} H_{0}$, generating space $\Theta_{i 0}^{\mathbf{B}^{i}}$ until the pellicle is reached, has to compensate the loss of actual energy (by the annihilation of the neutron). This is possible by generating a new major particle. Major point $P_{i}$ goes further inside $s^{0}$, until it coincides with $P_{0}$. Then space case 8 is reached, which for time case 1 (so $T_{i} \cap T_{0}=T_{i}$ ) generates a major particle (see equations (64) and (69)):

$$
Z\left(H_{i}^{*} H_{0}\right)=\left\{z_{1}, z_{2}\right\}=\left\{\begin{array}{l}
\left\{\left\{T_{i}, P_{i}, Q_{i}\right\},\left\{f^{i}, s^{i}, \partial \mathbf{E}_{i} / \partial t\right\}\right\} \\
{\left[\left\{F^{i} \backslash T_{i}, S^{i} \backslash P_{i}, \mathbf{B}^{i}\right\},\left\{\tau_{i}, \varnothing, \nabla \times \mathbf{B}^{i}\right\}\right]}
\end{array}\right\} .
$$

The mark term $\nabla \times \mathbf{B}^{i}$ is independent of space, so $\partial \mathbf{E}_{i} / \partial t$ as well; both may be replaced by a constant field $\mathbf{C}$. The appearance is:

$$
A\left(z_{1}, z_{2}\right)=\left\{\sigma_{s^{i}, Q_{i}},\left(\Theta_{S^{i} \backslash P_{i}}^{\mathbf{B}^{i}}\right)\right\} .
$$

This is a proton $\sigma_{i 0}^{+}$existing in the coinciding points $P_{i}$ and $P_{0}$, and an actual, unobservable, major extended space, written as $\Theta_{i 0}^{\mathbf{B}^{i}}$. Considering this together with step 2, the proton circles around the electron (and the neutrino still accompanies the electron).

Step 4: During the flying time, the three H-units have rearranged such, that $P_{j} \in p_{0}$ and $P_{i}=P_{0}$, so after the flying time, the total energy of the system can be written as:

$$
E_{2}\left(H_{i}^{*} H_{j} * H_{0}\right)=E\left(\left(e_{j 0}^{-}+v_{j 0}\right)+\left(\sigma_{i 0}^{+}+\Theta_{i 0}^{\mathbf{B}^{i}}\right)\right),
$$

in which the index 2 of $E$ indicates "after the interaction". A proton, an electron and a neutrino are generated; the magnetized space, existing at the start, appears again. The decay energy is still missing; in classical physics, this is supposed to be delivered by the kinetic energy of the electron. Because kinetic energy is related to velocity, and velocity is only possible in a space, we assume that according to twin physics, this energy can be found in a second zip (because this is the only one which may describe an extended space), in one of the three joint interactions.

To find this part of the decay process, we consider interaction $H_{i}{ }^{*} H_{j}$ again. In the beginning this generated the neutron with a gluon. After meeting the neutral pellicle, the neutron and the gluon annihilated and each of the marked H-units started a new career in combination with $H_{0}$, generating an electron with a neutrino and a proton, respectively. At the end of the decay story, the major points of space of $H_{i}$ and $H_{j}$ have a constant distance, equal to the radius of $s^{0}$, and they circle around each other. Because $s^{i}$ and $s^{j}$ are much smaller than $s^{0}$, their pellicles are supposed not to intersect.

Then, with $P_{i} \in S^{j}$ and $P_{j} \in S^{i}$, space case 6 is valid. If we take time case 2, for $T_{i}>T_{j}$, zipper $Z\left(H_{i}{ }^{*} H_{j}\right)$ contains three zips, being $z_{2}, z_{3}$ and $z_{4}$.

$$
Z\left(H_{i}^{*} H_{j}\right)=\left\{\begin{array}{l}
{\left[\left\{F^{i} \backslash T_{i} \cap F^{j}, S^{i} \backslash P_{i} \cap S^{j} \backslash P_{j}, \mathbf{B}^{i}+\mathbf{B}^{j}\right\},\left\{\tau_{i} \cap \tau_{j}, \varnothing, \widetilde{\nabla} \times \widetilde{\mathbf{B}}^{i} \propto \widetilde{\nabla} \times \widetilde{\mathbf{B}}^{j}\right\}\right]} \\
{\left[\left\{T_{i}, P_{i}, Q_{i}\right\},\left\{\varnothing, \varnothing, \partial \widetilde{\mathbf{E}}_{i} / \partial \tilde{t} \propto \widetilde{\nabla} \times \widetilde{\mathbf{B}}^{j} \propto \widetilde{\nabla} \times \widetilde{\mathbf{E}}_{i} \propto \partial \widetilde{\mathbf{B}}^{j} / \partial \tilde{t}\right\}\right]} \\
{\left[\left\{\varnothing, P_{j}, Q_{j}\right\},\left\{\varnothing, \varnothing, \partial \widetilde{\mathbf{E}}_{j} / \partial \tilde{t} \propto \widetilde{\nabla} \times \widetilde{\mathbf{B}}^{i} \propto \widetilde{\nabla} \times \widetilde{\mathbf{E}}_{j} \propto \partial \widetilde{\mathbf{B}}^{i} / \partial \tilde{t}\right\}\right]}
\end{array}\right\} .
$$

The appearance of the middle zip $\left(z_{3}\right)$ is a charged point particle $\Pi\left(P_{i}, Q_{i}\right)$ in $P_{i}$; its actual energy is equal to the actual energy of the charge. The last zip $\left(z_{4}\right)$ has no appearance, because both time elements are empty, but for $T_{i}<T_{j}$ the situation is mirrored and the appearance of this zip is a second charged point particle $\Pi\left(P_{j}, Q_{j}\right)$ in $P_{j}$. We suppose that these energies are already included in equations (83) and (87), so these appearances would double the amount of actual charge energy in equation (88) and thus they are not allowed. Possibly they play a role at the very moment of the annihilation of the neutron, which we did not consider in all its details.

The first zip $\left(z_{2}\right)$ describes a new item. Appearance $A\left(z_{2}\right)$ is an accelerated extended space, describing the turning around of the overlapping major spaces and thus the turning of $P_{i}$ and $P_{j}$ around each other; this will 
be written as $\Theta_{i j}^{(\mathbf{B})}$ (the upper index with round brackets indicates the changing magnetic fields). We identify this extended space energy with the kinetic energy of the electron.

Besides of this, it is experimentally known that in about one in 1000 decays an extra particle is produced, being a photon. Because a photon can only be generated by a mixed interaction, we will consider the mixed interaction products once again. If we consider $H_{j} * H_{0}$ not only in time case 4, as we did above, but also in time case 5, then $z_{2}$ in eq. (83) is the same, zip $z_{3}$ is empty and zip $z_{4}$ describes something new. For $T_{j}<T_{0}$ in time case 5 and space case 12 , zip $z_{4}$ is (see (78)):

$$
z_{4}\left(H_{j}^{*} H_{0}\right)=\left[\left\{T_{0}, P_{0}, \mathbf{b}^{j}\right\},\left\{\varnothing, \varnothing, \nabla \times \mathbf{B}^{j}+\partial \mathbf{B}^{j} / \partial t\right\}\right] .
$$

Appearance $A\left(z_{4}\right)$ is $\left(\Pi_{j 0}^{0}\right)$, being an unobservable, neutral, massless point particle, having a magnetic vector $\mathbf{b}^{i}$ attached and carrying an electromagnetic field vector of $\nabla \times \mathbf{B}^{j}+\partial \mathbf{B}^{j} / \partial t$.

This can be identified with a photon $\gamma_{j 0}$, traveling with a constant velocity around $P_{i}$ and carrying a part of the decay energy. Adding the energy of the photon and that of the extended space to equation (88), the resulting energy after the decay of the neutron can be written as:

$$
E_{2}\left(H_{i}^{*} H_{j}^{*} H_{0}\right)=E\left(\left(\Theta_{i j}^{(\mathbf{B})}\right)+\left(e_{j 0}^{-}+v_{j 0}+\gamma_{j o}\right)+\left(\sigma_{i 0}^{+}+\Theta_{i 0}^{\mathbf{B}^{i}}\right)\right) .
$$

This is supposed to be the complete description after the decay of the neutron, in case a photon is generated. Inserting the energies for the neutron $939.56 \mathrm{MeV}$, for the proton $938.27 \mathrm{MeV}$ and for the electron $0.51 \mathrm{MeV}$, with the decay energy of $0,78 \mathrm{MeV}$, the energy before the decay can be written as:

$$
E_{1}\left(H_{i}^{*} H_{j}^{*} H_{0}\right)=939.56 \mathrm{MeV}+E\left(\pi_{i j}\right)+E\left(\Theta_{i 0}^{\mathbf{B}^{\mathbf{i}}}\right) .
$$

and after the decay as:

$$
E_{2}\left(H_{i}^{*} H_{j}^{*} H_{0}\right)=E\left(\left(\Theta_{i j}^{(\mathbf{B})}\right)+\left(0.51+v_{j 0}+\gamma_{j o}\right)+\left(938.27+\Theta_{i 0}^{\mathbf{B}^{i}}\right)\right) .
$$

Actual energies (92) and (93) have to be equal. Supposing that the decay energy is equal to the actual energy of the extended space plus the eventually generated photon, we insert in equation (93):

$$
E\left(\Theta_{i j}^{(\mathbf{B})}\right)+E\left(\gamma_{j o}\right)=0.78 \mathrm{MeV}
$$

Then the energies of the gluon (before the decay) and the neutrino (after the decay) are related as:

$$
E\left(\pi_{i j}\right)=E\left(v_{j 0}\right) .
$$

Because the gluon (before the decay) and the neutrino (after the decay) have equal energies, the gluon is supposed not to annihilate together with the neutron, but to change its existence from 'bound to the neutron', into a 'free existence' in the neutral pellicle of the neutral H-unit, without being glued to a mass carrying particle.

Summarizing, a neutron generated by two oppositely marked H-units, having a gluon upon its surface, becomes unstable under the influence of the major space of a neutral H-unit. First it moves with a constant velocity towards the centre of this $\mathrm{H}$-unit. As soon as the neutron touches the neutral pellicle, it annihilates; the generation of its gluon is taken over from interaction $H_{i}{ }^{*} H_{j}$ to interaction $H_{j} * H_{0}$, and so the track of the gluon changes. Major point $P_{j}$, not fixed any more inside the neutron, sticks to the neutral pellicle; then, in one and the same new interaction, an electron is generated and the identity of the gluon is changed into a neutrino. Because a neutrino has a velocity close to that of light, we suppose that during the annihilation of the neutron, which is maximal the time necessary to cross the pellicle, the gluon accelerates to this velocity. After that, the positively marked H-unit, having crossed the neutral pellicle without sticking to it, enters the neutral minor space and goes further to the centre of the neutral minor space, to form the proton. The decay energy might be stored in an electromagnetic extended space representing the electron kinetic energy, or in an emitted photon, or both. To describe this phenomenon, we need three H-units and all four zips, to derive three zippers in several time and space cases.

It would be interesting to investigate if a hydrogen atom can be described by the interaction of four $\mathrm{H}$-units. 


\section{Comparison with the Standard Model}

The Standard Model is a theory of particle physics, classifying all subatomic particles known, as well as concerning the electromagnetic, weak, and strong nuclear interactions. It was developed throughout the latter half of the 20th century by collaborating scientists around the world, theoretical and experimental particle physicists alike. The current formulation was finalized in the mid-1970s upon experimental confirmation of the existence of quarks. The discoveries of the top quark (1995), the tau neutrino (2000), and the Higgs boson (2012) have given further credence to the Standard Model. For theorists, the Standard Model is a paradigm of a quantum field theory, which exhibits a wide range of physics including spontaneous symmetry breaking, anomalies and non-perturbative behavior.

However, the Standard Model leaves some phenomena unexplained and it falls short of being a complete theory of fundamental interactions; it does not incorporate the full theory of gravitation as described by general relativity and it does not account for the accelerating expansion of the universe.

The most fundamental concept of the Standard Model is, that the universe is built up in a deterministic way. Determinism is still strongly defended by many physicists, for instance by Rietdijk (2003), who used this concept to construct a theory about hidden variables. However, there are signs of indeterminism pressing onward, like in the recent paper of Langley (2016, section 8.1), who needs 'true randomness' in his theory about gravity; this can be conceived as a notion of indeterminism. Possibly even the incompatibility of Einstein's relativity theory with quantum mechanics is related to its deterministic basis.

By contrast, twin physics is based upon the concept that the world is dualistic; determinism and indeterminism are considered as intimately connected. To be able to describe indeterminate objects independently of deterministic ones, a mathematical language called 'complementary language', based upon set theory, has been developed. Spaces are considered as limited, energetic items; also the Heisenberg's uncertainty relations are translated into a dual description. An important difference is, that the concept of 'force' is not represented in twin physics; energetic space supplies a substitution for forces.

The basic element in twin physics is the H-unit, being a mathematical unity of potential energy; this is strongly related to the original conception of quarks, introduced by Gell-Mann (1964), in which he distinguished mathematical and physical quarks, the former being mathematical expedients. Quarks might be the deterministic precursors of H-units. An important difference between the Standard Model and twin physics is in conceiving potential energy; in twin physics, potential energy is not a real energy which can be assigned to a body, but an intermediate mathematical item.

In this paper, we described with one set of four elements (the zipper), containing entangled attributes of time, space and charge, all basic phenomena in physics, like the neutron, the proton and its anti-particle, the electron and its anti-particle, the gluon, the neutrino, the photon, the Higgs particle, including spin (Backerra, 2012). Moreover, the laws of Maxwell emerge from the definitions; gravity can be connected to electric attraction and repulsion; the phenomena expansion of the universe, the weak force and the strong force can be described in principle. The constant velocity of light and the quantisation of Planck can be deduced (Backerra, 2015), instead of taking them as postulates.

\section{Conclusion}

According to twin physics, the range of gravity is larger than that of electricity. This is related to the restriction of spaces to finite sizes, combined with an essential difference in size between neutral and marked H-units. Marked H-units have to spend a part of their potential energy to charges and fields, so not all potential energy is available for space. This difference in ranges might explain the instability in the experimentally observed constants of gravity (Zyga, 2015). The type of interaction between H-units which is identified with gravity not only leads to attraction but may also generate repulsion. Because this latter phenomenon occurs only beyond the reach of electromagnetic influences, expansion of the universe is unjustly considered to be an independent phenomenon.

The decay of a free neutron can be described using three H-units. Although the zipper in general describes only the interactions between two $\mathrm{H}$-units, it seems possible to describe this phenomenon properly by considering the three interaction possibilities of the H-units separately and using the law of conservation of actual energy to connect them. The decay of the neutron, described by two marked H-units, is initialized if a third, neutral H-unit crosses its space. At that moment the marked H-units can no longer spend all their potential energy on the previous type of interaction, so the $\mathrm{H}$-units will rearrange themselves to generate a proton, an electron, a neutrino and decay energy. This explains in principle why the moment of decay for a single neutron is unpredictable; the 
average time of decay might give insight into the proportions of the universe. The description predicts that upon a free neutron a gluon exists, which during the decay transforms into a neutrino.

An important feature of twin physics is the formulation of one zipper for all phenomena. Heisenberg uncertainty relationships, relativity, and small-scale as well as large-scale observations are incorporated within the zipper. The use of four-dimensional space-time descriptions, being difficult to imagine, is not necessary and is even undesirable. As in the Standard Model, the elementary particles - neutron, proton, electron, gluon, neutrino, Higgs particle and photon - can be described. In twin physics, dark matter can also be described and the existence of an anti-photon is predicted. Not all experimentally found variants of elementary particles have been considered yet. The quark is considered to be the deterministic precursor of the complementary H-unit.

In contrast to the Standard Model, twin physics makes no use of the concept of forces. This is made possible by considering finite spaces as independent energetic items, connecting particles. The strong force is supplied by the minor space of neutral H-units. The weak force is represented by a rearrangement of $\mathrm{H}$-units resulting from their constant potential energy in combination with the law of conservation of actual energy. The electromagnetic force is supplied by coupling appropriate features to geometric features of H-units. The laws of Maxwell are not plugged in, but emerge from these definitions. The gravitational field has its analogue in twin physics by taking interacting neutral $\mathrm{H}$-units as a background for electromagnetic sensible mass; the possibility of expansion as a second feature of the same interaction pops up.

While quantum mechanics and astronomy are not compatible in the Standard Model, in twin physics small- and large-scale physics are reconciled from the start. The constancy of the velocity of light and the quantization of the photon energy can be formally deduced in twin physics. This strengthens the supposition that twin physics may cover the complete field in an undivided way.

For the time being, the disadvantage of twin theory lies in the restriction of the system used to only a few H-units. The zipper describes the interaction of two H-units only and it seems appropriate to take a few more into consideration by incorporating the energetic balance. The description of systems containing a large number of H-units needs to be developed.

The major disadvantage of the Standard Model is the incompatibility of its constituent parts. The view of Einstein's relativity theory as an unimpeachable theory is at least open to question. The relatively large attention to the deterministic aspects of physics may be unjustified.

\section{Acknowledgments}

I owe the conceptual idea for this paper to Willem van Erk, who also assisted me in correcting the paper. I am very grateful to Wim Graef, who challenged me to defend the principles of twin theory in a series of fundamental discussions. Special thanks to the Taurida International Symphony Orchestra in Saint Petersburg (Russia), under the guidance of Mikhail Golikov, for allowing me to work on this subject while attending their rehearsals and concerts. Their music provided me with inspiration that I could make full use of thanks to their warm hearted reception, which turned Russia into my second homeland.

\section{References}

Backerra, A. C. M. (2010). Uncertainty as a principle. Physics Essays, 23(3), 419-441.

Backerra, A. C. M. (2012). The unification of elementary particles. Physics Essays, 25(4), 601-619.

Backerra, A. C. M. (2014). The quantum-mechanical foundations of gravity. Physics Essays, 27(3), 380-397.

Backerra, A. C. M. (2015). A bridge between quantum mechanics and astronomy. Applied Physics Research, 8(1).

Gell-Mann, M. (1964). A Schematic Model of Baryons and Mesons. Physics Letters, 8 (3): 214-215

Hudson, J. J., Kara, D. M., Smallman, I. J., Sauer, B. E., Tarbutt, M. R., \& Hinds, E. A. (2011). Improved measurement of the shape of the electron. Nature, 473(7348), 493-496.

Kahn, P. J. (1967). Introduction to linear algebra. London: Harper \& Row, Ltd.

Langley, R. (2016). Quantum Gravity as Higher Dimensional Perspective. Applied Physics Research, 8(4).

Michelson, A. A., \& Morley, E. W. (1887). On the Relative Motion of the Earth and the Luminiferous Ether. American Journal of Science, 34, 333-345.

Newton, I. (1687). The mathematical principles of natural philosophy (or: Principia), page 77. Retrieved from http://docs.lib.noaa.gov/rescue/Rarebook_treasures/QA803A451846.PDF

Rankine, W. J. (1853). On the general law of the transformation of energy. Philosophical Magazine, 4(5), 106-117. 
Rietdijk, C. W. (2003). How do hidden variables fit in natural law? Physics Essays, 16(1), 42-62.

Weinberg, S. (2015). To explain the world. Great Brittain: Allen Lane.

Zyga, L. (2015). Why do measurements of the gravitational constant vary so much? Retrieved from http://phys.org/news/2015-04-gravitational-constant-vary.html\#jCp

\section{Copyrights}

Copyright for this article is retained by the author(s), with first publication rights granted to the journal.

This is an open-access article distributed under the terms and conditions of the Creative Commons Attribution license (http://creativecommons.org/licenses/by/4.0/). 